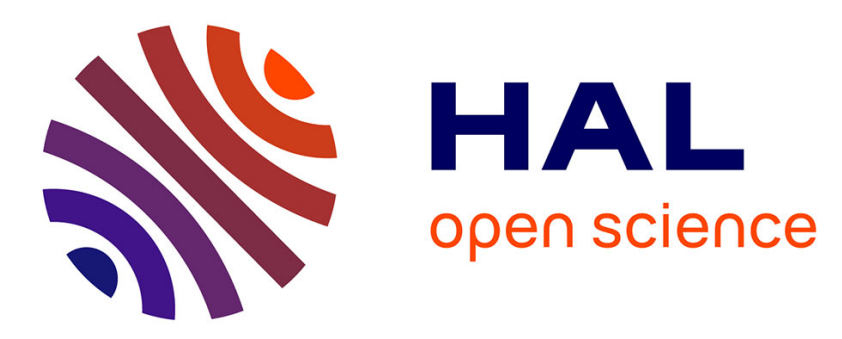

\title{
Numerical simulation of fenestrated graft deployment: Anticipation of stent graft and vascular structure adequacy.
}

Claire Dupont, Adrien Kaladji, Michel Rochette, Blandine Saudreau, Antoine Lucas, Pascal Haigron

\section{To cite this version:}

Claire Dupont, Adrien Kaladji, Michel Rochette, Blandine Saudreau, Antoine Lucas, et al.. Numerical simulation of fenestrated graft deployment: Anticipation of stent graft and vascular structure adequacy.. International Journal for Numerical Methods in Biomedical Engineering, 2021, 37 (1), pp.e03409. 10.1002/cnm.3409 . hal-02998720

\section{HAL Id: hal-02998720 \\ https://hal.science/hal-02998720}

Submitted on 18 Dec 2020

HAL is a multi-disciplinary open access archive for the deposit and dissemination of scientific research documents, whether they are published or not. The documents may come from teaching and research institutions in France or abroad, or from public or private research centers.
L'archive ouverte pluridisciplinaire HAL, est destinée au dépôt et à la diffusion de documents scientifiques de niveau recherche, publiés ou non, émanant des établissements d'enseignement et de recherche français ou étrangers, des laboratoires publics ou privés. 


\title{
Numerical simulation of fenestrated graft deployment: anticipation of stent graft and vascular structure adequacy
}

\author{
Claire Dupont ${ }^{1 *}$, Adrien Kaladji ${ }^{1}$, Michel Rochette ${ }^{2}$, Blandine Saudreau ${ }^{3}$, Antoine Lucas ${ }^{1}$, and Pascal \\ Haigron $^{1}$
}

${ }^{1}$ Univ Rennes, CHU Rennes, Inserm, LTSI - UMR 1099, F-35000 Rennes, France

${ }^{2}$ Ansys France, F-69100 Villeurbanne, France

${ }^{3} \mathrm{CHU}$ Rennes, F-35000 Rennes, France

\begin{abstract}
Fenestrated endovascular aneurism repair (FEVAR) is a minimally invasive technique, and its success depends on the adequacy of the correspondence between the visceral arteries ostia and position of the fenestrations of the stent graft (SG) during its deployment in juxtarenal aneurisms. However, the fenestration position is generally determined from a preoperative computerised tomography (CT) scan, without considering the vascular deformation induced by the insertion of the endovascular tools. Catheterisation difficulties may occur during clinical procedures. Accordingly, the objective of this work is to present an initial proof of concept aimed at anticipating and optimising the position of the fenestrations, while considering the vascular deformation induced by the insertion of the endovascular tools. The proposed method relies on the finite element method to simulate the SG deployment in a vascular structure (VS), and considers the vascular deformation induced by the tools. After determining the optimal simulation parameters for a patient-specific case, the robustness of the method is demonstrated on six other representative anatomies. The simulated SG is also compared with post-deployment CT observations, and demonstrates good adequacy. The results show that the numerically corrected fenestration positions, as determined from the simulated results following the insertion of the endovascular tools, deviate from those of the standard plan (as determined from the preoperative CT scan). This indicates that the SG-VS adequacy could be improved via simulation-based planning, to anticipate potential catheterisation difficulties.
\end{abstract}

\section{Introduction}

The number of abdominal aortic aneurisms treated with endovascular aneurism repair (EVAR) is increasing annually. Initially reserved for patients contraindicated for open surgery, this minimally invasive treatment is a safe and effective alternative treatment, which augment the therapeutic options and made it possible to treat more patients [1]. This procedure involves excluding the aneurysmal sac by introducing one or more stent grafts (SGs) via the femoral arteries.

However, nearly $15 \%$ of patients have short necks, that is, the length of the healthy aorta between the renal arteries and beginning of the aneurism sac is less than $10 \mathrm{~mm}$ [2]. This vascular segment (corresponding to the SG sealing area in conventional EVAR procedures) is extremely short, making it difficult to ensure sufficient sealing and avoid endoleaks. In such cases, a fenestrated SG with lateral fenestrations can be deployed above the visceral arteries (renal arteries, superior mesenteric artery, and celiac trunk). Procedures involving this type of fenestrated (FEVAR) SGs require the alignment of the fenestrations with the ostia of the visceral arteries, thus increasing the complexity. Incorrect fenestration positioning increases the duration of the procedure, anaesthesia, and fluoroscopic exposure, and sometimes requires conversion to open surgery. The 30-day follow-up for fenestrated EVARs shows a mortality rate of $3-5 \%$ and a morbidity rate of $12-40 \%$, including mesenteric ischaemia and renal complications [3]-[5].

The appropriate planning of fenestration positioning is important for achieving good long-term results [6][8]. However, Maurel et al. [9] observed a significant displacement of the visceral ostia following the insertion of endovascular tools (such as catheters, stiff guidewires, and delivery systems). As the positions of the fenestrations on a standard fenestrated SG plan are defined based on a preoperative computed 
tomography (CT) scan, their displacements relative to the intraoperative anatomical deformations are not considered. In cases where significant displacement is observed, it is difficult for the surgeon to catheterise all the visceral arteries during the endovascular procedure, resulting in potential subsequent complications.

To improve the planning of the endovascular procedure, physical phantoms of the aorto bi-iliac structures can be created using 3D printing [10], [11]. For example, Terumo Aortic included a step in their planning during which a prototype of the Anaconda prothesis was test implanted in a printed silicon model of a patient's aorta. Surgeons and engineers could reproduce the endovascular procedure in silico and review their planning (which was initially performed on a preoperative CT scan). [12] showed that approximately $20 \%$ of the prototypes of the fenestrated SG were changed after this test implantation. However, the use of physical phantoms to plan interventions under realistic conditions is time-consuming. Recently, Starnes et al. [13] created fenestrated SGs using a 3D printing template, to locate the position of the fenestrations on the SGs. To position the fenestrations, the vascular structure centreline and visceral arteries ostia were extracted from the preoperative $\mathrm{CT}$. The initially curved centreline was then artificially straightened using a dedicated algorithm. The ostia were then projected onto a straight cylindrical template created from the straightened centreline. Subsequently, the surgeon cut the fenestrations on the SG. Even though this technique appears promising in cases of emergencies, it does not consider the real deformations between the vascular structure and SG.

The development of biomechanical numerical models is a promising alternative for accurately anticipating the adequacy, that is, the alignment of the visceral arteries with the SG fenestrations, as well as the interactions between the medical devices (endovascular tools and implanted SGs) and vascular structures. The preservation of the visceral arteries is closely linked to catheterisation difficulties. It is difficult to anticipate these challenges based only on image analysis, owing to the deformability of the anatomical structures. In recent years, models based on the finite element method have been proposed to study the interactions between tools and vascular structures during endovascular procedures [14]-[17]. Gindre et al. [16] developed a method for simulating the aorto bi-iliac structure deformation induced by the insertion of stiff guidewires during EVAR, without simulating the SG deployment. Recently, Mohammadi et al. [18] incorporated bones, surrounding tissues, and the intraluminal thrombus when modelling the insertion of endovascular tools. The finite element method was also employed to simulate the SG deployment. Morphing methods were developed to identify the deployed SG configuration in a synthetic geometry [19], or to identify non-deformable aortoiliac structures from pre-operative CT images [17]. Simulations of SG deployment integrating anatomical deformations have been reported for synthetic arteries [20] or patientspecific geometries [14], but neglect the vascular deformations induced by the insertion of endovascular tools. Recently, Sanford et al. [21] modelled the deformation of a vascular structure from stiff guidewires. They subsequently modelled the insertion of a delivery system, in which a SG was compressed before being deployed at the desired location. In particular, they focused on the potential for SG rotation following its deployment, and during removal of stiff guidewires and delivery systems. Certain parameters that might affect the resulting vascular deformation, SG deployment after release, and/or residual rotation, such as bone support or calcifications, have not been considered [22]. Although rotation could potentially modify the final alignment of the fenestrations, anticipating the adequacy between the fenestrated SG and vascular structure during catheterisation of the visceral ostia remains challenging. The objective of this study is to define a numerically corrected plan for the fenestrated SG, considering the vascular deformation induced by the insertion of the endovascular tools.

To reduce the risk of intraoperative complications such as catheterisation difficulties or incorrect perfusions of the visceral arteries, this article presents a numerical method for simulating a FEVAR procedure, and anticipating the position of the SG fenestrations during delivery. The initial proof of concept is supported by preliminary clinical data. Following a synthetic presentation of the endovascular procedure, the 
proposed finite element method used to simulate the SG deployment, and the method employed to determine the corrected plan from the simulation results are described in Section 2 . The parameter settings for the algorithm and adequacy between the SG and vascular structure are presented in Section 3 . The results of this study are discussed in Section 4.

\section{Methods}

\subsection{Patients' data}

The data were collected retrospectively at Rennes University Hospital, from seven patients who underwent consecutive FEVAR procedures between mid-2016 and mid-2017. The protocol and informed consent form were approved by the local ethics committee, and all subjects provided informed consent (French National Agency regulating Data Protection declaration $n^{\circ}$ 1932135, Feb 2016). All patients who underwent FEVAR during the protocol period were included. Six male and one female patients were enrolled, with a mean age of 82.3 years. The median body mass index was 24.5. One patient presented a type IV thoracoabdominal aneurism, one patient had an inter-renal aneurism, and five patients had a juxtarenal aneurism. The mean diameter of the aneurisms was $60 \mathrm{~mm}$. All of the implanted SGs were designed and manufactured by Cook Medical Company (William Cook Europe, Biaeverskow, Denmark). The surgeon had the responsibility of the graft plan and checked each measurement; however, this was carried out without the help of simulation at the time of the procedure. All patients underwent at least one postoperative CTscan at 7 or 30 days after discharge.

\subsection{Endovascular treatment with fenestrated stent graft (SG)}

Endovascular treatment begins with a sizing step. This pre-operative planning step is essential to ensuring that the endovascular treatment proceeds smoothly. During this phase, the surgeons select SGs suitable for the patient's anatomy, depending on the diameter of the aorta measured at the neck, diameter of the aneurism, and iliac arteries. Furthermore, the length of the proximal aortic neck and length between the lowest renal artery and bifurcation are also considered [23]. In the case of FEVARs, the exact position of the ostia is also identified in a preoperative CT scan. The fenestrated SG (considered a 'custom-made device') can be constructed based on the above measurements and ostia localisation. The bifurcated SG and limbs, which are subsequently deployed to ensure watertightness with the vascular wall, are then selected from the available references. Oversizing (10-20\%) is always applied to the measured diameters, to avoid endoleaks.

The analysis of the preoperative CT scan also allows the surgeons to determine the introduction side for the fenestrated and bifurcated SG, based on the calcification rate and tortuosity of the iliac arteries.

The main steps employed to deploy the fenestrated SGs are summarised as follows. After puncturing the common femoral arteries, a flexible guidewire was inserted up to the ascending aorta under fluoroscopic control. It was replaced with a stiff Lunderquist guidewire through a catheter previously inserted around the flexible guidewire. The delivery system, in which the fenestrated SG was compressed, was then inserted into the stiff guidewire. The fenestrations, identified by radiopaque markers, were positioned with respect to the ostia using fluoroscopy. The fenestrated SG was deployed to $2 / 3$ of its circumference. Each fenestration was catheterised using a flexible guidewire, and was subsequently replaced by a stiff guidewire introduced into the contralateral femoral artery. This step required the correct positioning of all of the fenestrations in front of the ostia. The stents were positioned through the fenestrations before the total deployment of the fenestrated SG. The introduction and inflation of a balloon ensured that the proximal part of the fenestrated SG was in contact with the vascular wall (to avoid endoleaks). The covered stents were then deployed in the visceral arteries and riveted with a balloon. The bifurcated SG, compressed in 
another delivery system, was then introduced up to the appropriate radiopaque markers before being deployed. The limbs were then deployed in each of the iliac arteries.

\subsection{Simulation process}

In this study, the deployment of a fenestrated SG is simulated, without reproducing all of the steps of the classic clinical procedure described in Section 2.1. Only the insertions of endovascular tools with a significant influence on vascular deformation were simulated, so as to simplify the problem. The numerical workflow is illustrated in

Figure 1, and the biomechanical model is described in detail in Section 2.4.

With respect to the clinical procedure, the numerical workflow began by analysing the preoperative CT scan to identify the visceral and internal iliac ostia, and to extract the aortoiliac geometry and parameters necessary to define the boundary conditions. These data were then used to simulate the vascular deformations induced by the insertion of the catheters, stiff guidewires in the two iliac arteries, and insertion of the delivery system in the iliac artery as selected by the surgeon during the sizing process. Once these tools were inserted, the SG was compressed into the deformed artery. The contacts between the SG and vascular structure were then activated, and the SG was released into the aorta. The endovascular tools were removed in the reverse order in which they were introduced. The deformed configuration of the vascular structure and fenestrated SG could subsequently be analysed and evaluated, to determine a numerically corrected plan for the fenestrated SG. 


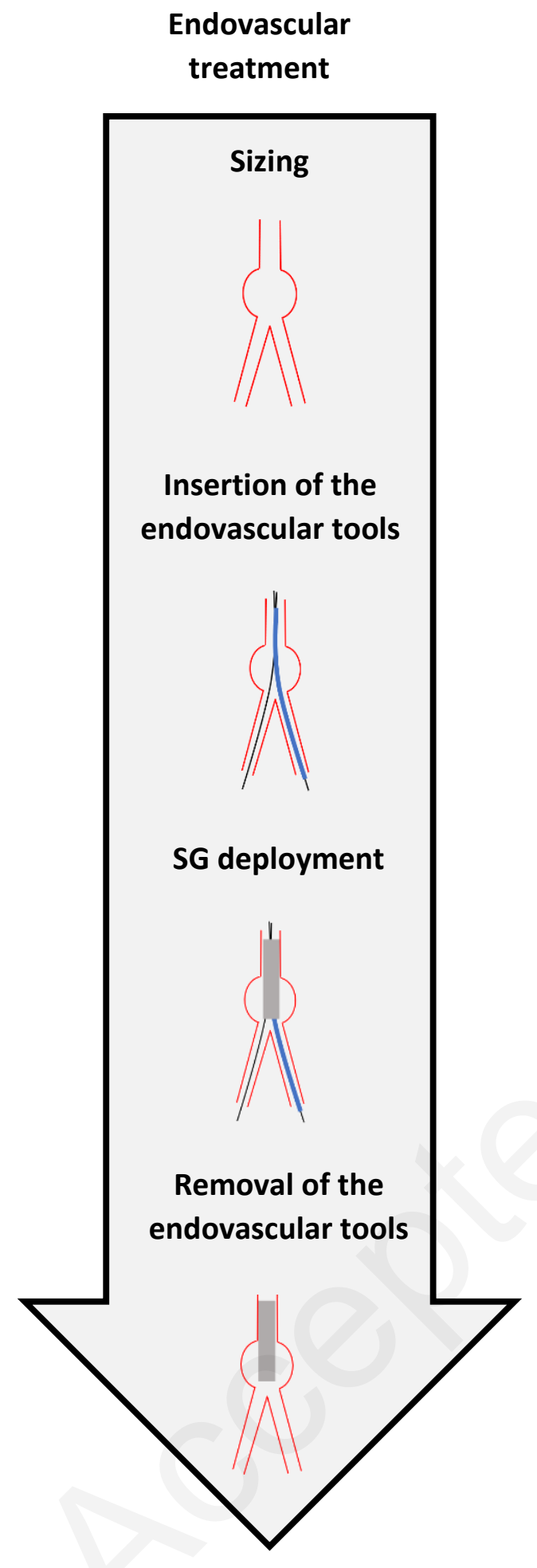

\section{Numerical workflow}

\section{Preoperative CT scan}

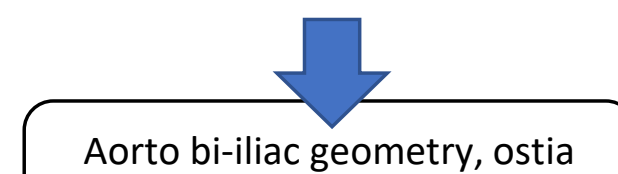

position, boundary conditions

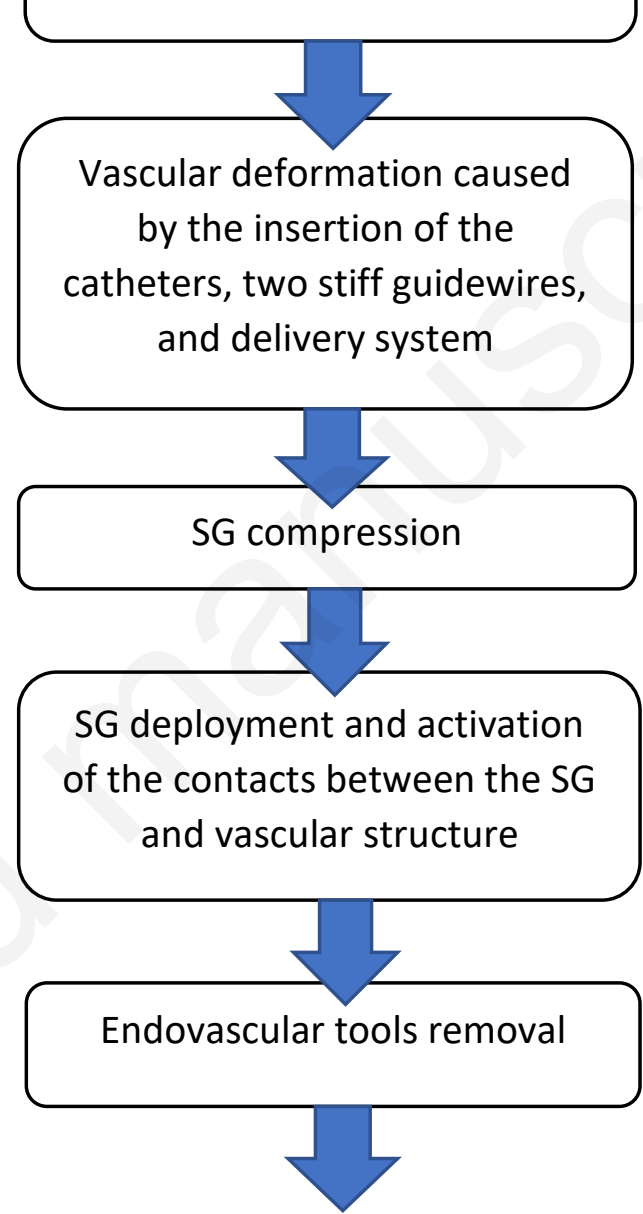

Numerically corrected plan of the fenestrated SG

Figure 1: Illustration of the main steps of the clinical treatment in parallel with the numerical workflow

\subsection{Biomechanical model}

2.4.1. Vascular deformation modelling 
The reference vascular mesh, centre position of the visceral collateral arteries, and data required to define the boundary conditions were extracted from the pre-operative CT scan, using the sizing software Endosize ${ }^{\circledR}$ (Therenva, France). This software is employed by surgeons in clinical routines to extract centrelines and measure characteristic diameters or lengths. As the centres of the collateral arteries identified on the pre-operative CT scan could be located outside the vascular mesh, these points were projected onto the mesh, to find the closest nodes. The ostia centres were then represented by these nodes, and their displacements were followed during the simulations.

The geometry extracted from the preoperative CT scan was subject to blood pressure. A preliminary step of the simulation involved determining the 'blood pressure free' geometry, which, once subjected to blood pressure, corresponds to the geometry extracted from the pre-operative CT scan. The method developed by Gindre et al. [14] was used to estimate the 'blood pressure free' geometry, and to simulate the insertion of the endovascular tools. The mechanical properties of catheters and stiff guidewires conventionally handled during the endovascular procedure were considered. The mechanical properties of the delivery system represented the case where a SG is compressed inside.

The 'blood pressure free' geometry, pressurisation of this geometry, and insertion of the endovascular tools were determined using the LS-dyna 16 explicit software, according to the method developed by Gindre et al. [14] (Ansys, Canonsburg, Pennsylvania, USA). The methods used to create the vascular structure, determine the boundary conditions, represent the support of the surrounding tissues (e.g. bone structures and hypogastric collateral), and insert the endovascular tools are summarised in Appendix $A$. The mechanical properties of the endovascular tools are summarised in Appendix B.

\subsubsection{SG modelling}

A SG consists of a stent, i.e. a cylindrical wire mesh that is used as a mechanical support and is sewn onto a semi-permeable tissue called a graft to guide blood flow, exclude aneurisms from blood circulation, and avoid ruptures of the vascular wall.

In this study, a clinically used fenestrated SG (Cook Medical ${ }^{\circledR}$ ) was considered. This SG is described by its centreline $C L_{S G}$ and radius $R_{0}(\alpha)$. The point $P_{\text {deploy, }}$ defined as the centre of the circular section formed by the distal end of the graft, represents the origin of $C L_{S G}$. $C L_{S G}$ can be parameterised by its curvilinear abscissa $\alpha$. $R_{0}(\alpha)$ varies along the $C L_{S G}$ on the standard plan of the fenestrated SG as illustrated in Figure $2 \mathrm{a}$, as determined from the preoperative $C T$ scan considering $C L_{S G}$ as a straight line. The fenestrated SG surface can be described by a set of points generated owing to the displacement of a circle with radius $R_{0}(\alpha)$ along the centreline $C L_{s G}$. In the reference frame $(\vec{n}(\alpha), \vec{b}(\alpha), \vec{t}(\alpha))$ associated with the centreline $C L_{s G}$, the coordinates of the points describing the SG surface can be defined using the following parametric equation:

$$
S_{S G}(\alpha, \theta)=\gamma(\alpha)+R_{0}(\alpha)[-\cos (\theta) \vec{n}+\sin (\theta) \vec{b}][24],
$$

Eq. 1

where $\gamma(\alpha)$ represents the centre coordinates of a circular section with a curvilinear abscissa $\alpha, \vec{n}$ is the vector normal to $C L_{S G}, \vec{b}$ is the binormal vector to $C L_{S G}$, and $\vec{t}$ is the vector tangent to $C L_{S G}$. In the plan defined by $\vec{b}(\alpha)$ and $\vec{t}(\alpha), \theta$ is the angle between $\vec{b}$ and a vector determined by the SG surface point and a point on $C L_{s G}$. The graft mesh can be created by sampling the SG centreline so that the circular sections pass through the ends of each stent. The intermediate circular sections are also described, to limit convergence problems related to graft folding. As the SG wire mesh consists of several sinusoidal stents, the position of the centre of the circular section along the centreline $C L_{S G}$ can be defined as follows:

$$
\gamma(\alpha)=\frac{H_{S i}}{2} \sin \left(\theta N_{P}\right)+\sum_{k=0}^{i-1} H_{S_{k}}+(i-1) H_{G}
$$


where, $N_{p}$ is the number of stent patterns, i.e. the number of sinusoids corresponding to each stent, $H_{s i}$ is the height of the stent of interest $i$, and $H_{G}$ is the constant spacing between the stents. Quadrilateral elements are obtained by connecting the points. Their size depends on the sampling steps of $\alpha$ and $\theta$. The graft mesh is based on the sinusoidal shape of the stents. To facilitate graft folding, the quadrilateral elements are cut along one of their diagonals. As the $0.02 \mathrm{~mm}$ graft thickness is lower than the other graft dimensions, it is modelled using triangular shell elements. The node coordinates of the ends of the beam elements used to model the stents can be derived from Eq. 1 and 2. In this manner, a few nodes of the graft coincide with those of the stents. Thus, contact between the beam and shell elements is not necessary to model the stitches between the graft and stents. No fenestrations are represented on the graft mesh. The mesh element size is determined empirically to achieve a trade-off between the computation time and degree of accuracy required for displacement estimation. An example of the initial SG mesh is shown in Figure $2 \mathrm{~b}$, as modelled with 1319 beam elements and 23759 shell elements, when $C L_{S G}$ is a straight line.

The stents were composed of Nitinol. This biocompatible shape-memory alloy allows for high compression of the fenestrated SG in a delivery system and a spontaneous return to its original shape (e.g. in response to a decrease in the mechanical stress and/or temperature variations). The graft is assumed to be composed of polyethylene terephthalate, known as Dacron ${ }^{\circledR}$. The superelastic behaviour of Nitinol was modelled using the Auricchio and Taylor model [25]. The mechanical properties considered while modelling the graft and stents can be characterised as mentioned in [26] and [27], respectively, and are detailed in Appendix B.

a)

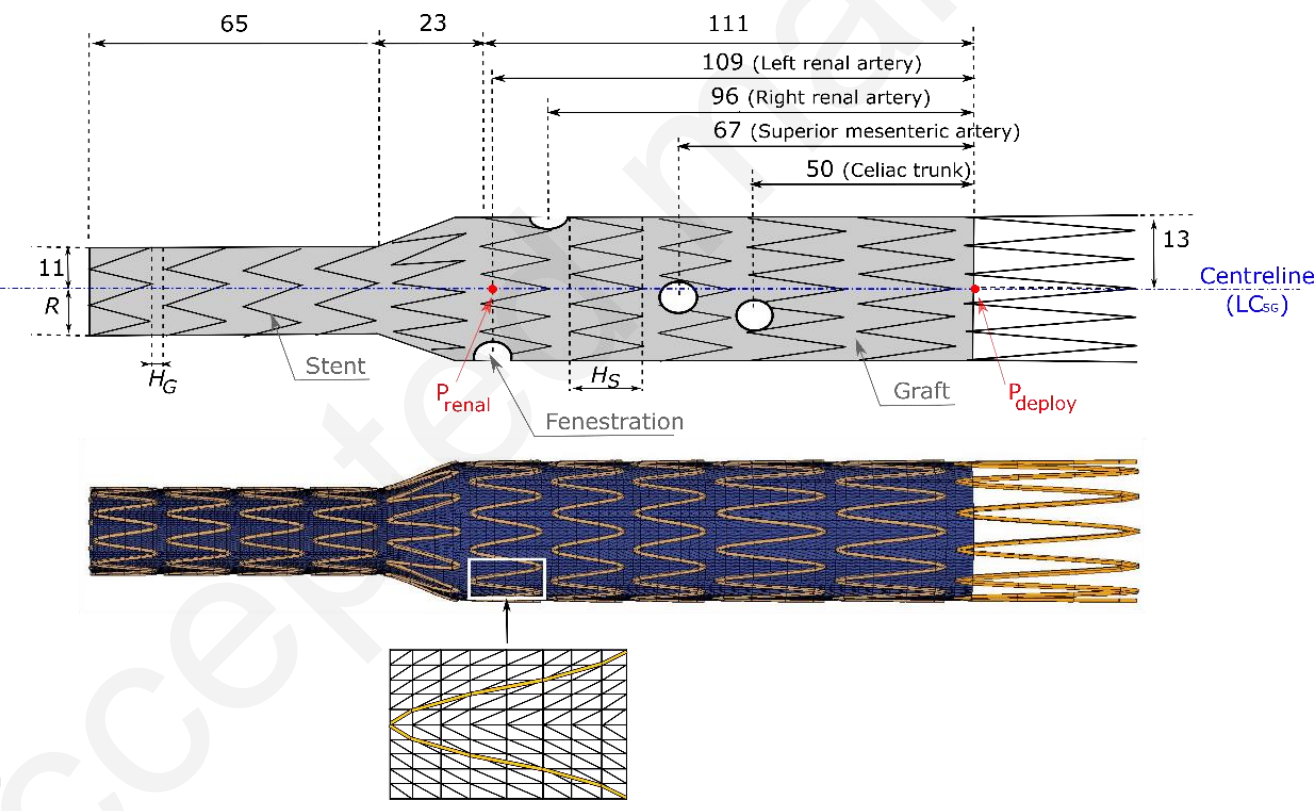

Figure 2 : Example of the a) standard plan of an implanted $S G$ and b) corresponding mesh when $C L_{S G}$ is a straight line

\subsubsection{Determination of the aligned and compressed geometries of the SG}

In the proposed approach, the SG was not compressed into the delivery system. The deployment simulation was based on the preliminary determination of the SG geometry, which was aligned with the aorta centreline. During an endovascular procedure, the SG centreline is not straight at the time of deployment; however, it corresponds to that of the delivery system. However, the delivery system might be tangential to the vascular wall. An initialisation of the compressed SG on the axis of the delivery system requires a 
large compression of the SG, resulting in numerical instabilities related to the graft folds in contact with each other. The SG centreline was aligned with one of the vascular structures.

To overcome the numerical instabilities, the compressed geometry obtained after reducing the radius was then imposed, i.e. to artificially compress the SG once the delivery system was inserted into the vascular structure (the SG centreline remained unchanged). The SG was subsequently released. This method dissociated the effects of SG compression from those of SG deformation owing to the insertion of the delivery system into a tortuous vascular structure. The method used to determine the aligned and compressed geometries of the SG is illustrated in Figure 3.

Numerically, the SG was aligned with the centreline of the vascular structure, $C L_{v s \_d e f}$, as deformed by the stiff guidewires and delivery system. This latter parameter was calculated from the surface mesh describing the deformed vascular structure using the open source code Vascular Modelling ToolKit (VMTK,) [28]. The maximum radius of the sphere inscribed in the vascular structure $R_{\text {def }}(\alpha)$ was also extracted along the centreline $\mathrm{CL}_{\text {vs_def. }}$

The determination of the initial SG geometry involved maintaining its initial radius $R_{0}(\alpha)$ and aligning its centreline $C L_{s G}$ with the centreline $C L_{v s_{-} \text {def }}$ from the reference point $P_{\text {deploy }}($ Eq. 1,2$)$. Similarly, the geometry of the compressed SG was calculated by replacing the radius $R_{0}(\alpha)$ with $C \times R_{d e f}(\alpha)$ ( $C$ is a compression factor), so that the compressed SG was fully inscribed in the vascular structure. This solution permitted the compression (pre-deformation) of the stents, to reproduce the interactions between the SG and vascular wall during deployment [29]. 


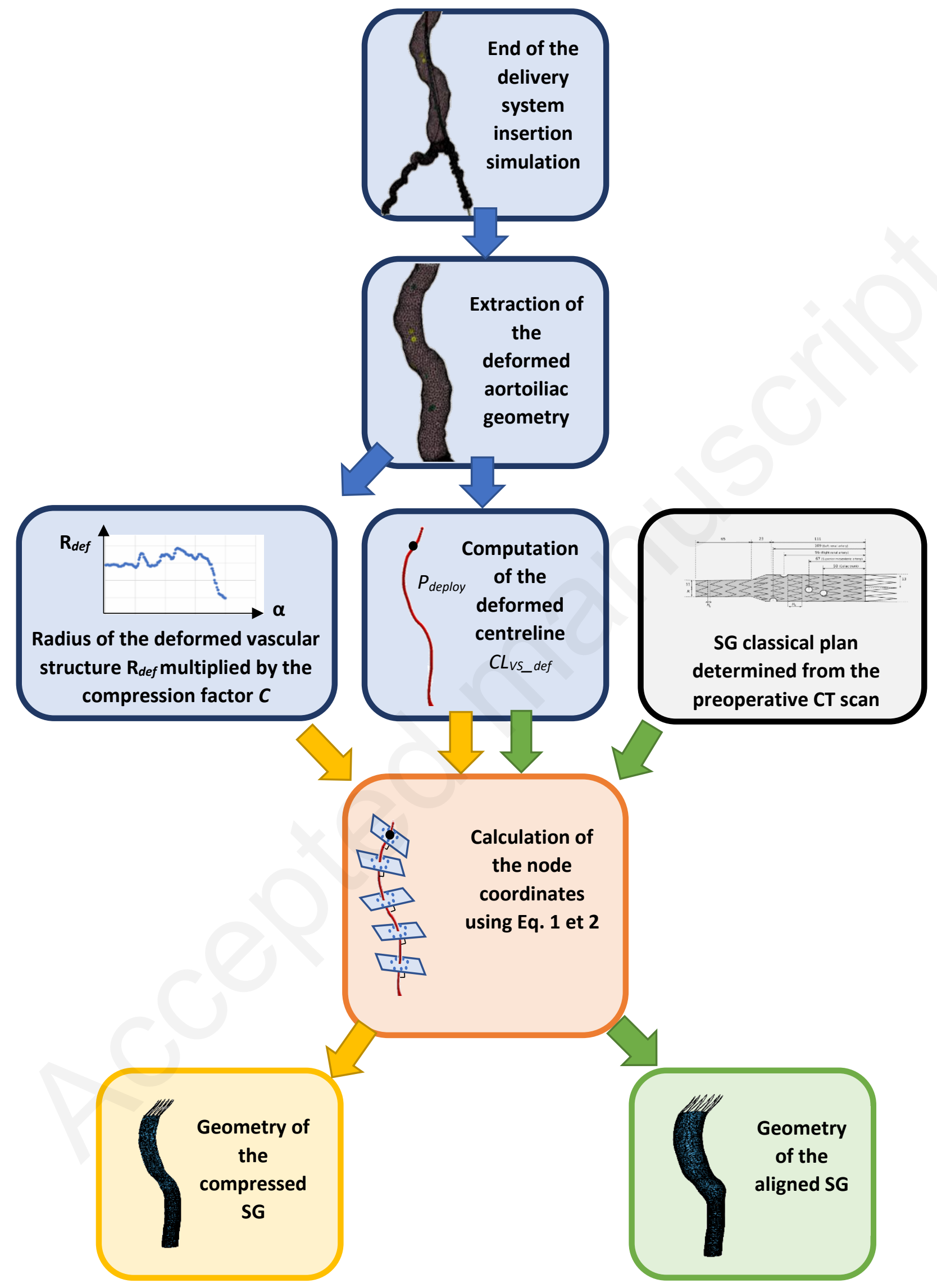

Figure 3: Method used to compute the aligned and compressed SG geometries 


\subsubsection{Simulation of SG deployment and endovascular tools removal}

Once the delivery system was inserted, the SG was compressed into the deformed aorto bi-iliac structure, by imposing only the position of the stent nodes from the previously determined compressed SG geometry. This compression step, controlled by $C$, allowed the SG to be fully inscribed in the aortic lumen while minimising graft folding.

When the SG was fully compressed, the constraints on the position of the stent nodes were removed. The SG unfolded spontaneously. The timing of this spontaneous deployment step was such that the time interval $\Delta t$ between the removal of the constraints on the position of the stent nodes and start of removal of the endovascular tools (stiff guidewires and delivery system) allowed the SG to reach its maximum deployment.

The endovascular tools were then removed by imposing a velocity at their distal end, directed outward from the vascular structure. They were extracted from the vascular structure in the reverse order in which they were introduced, i.e. the delivery system, then the stiff guidewires, and finally the catheters.

To limit the SG vibrations during deployment, Rayleigh damping proportional to the mass of the beam elements modelling the stents was introduced. A proportionality function was determined such that the compressed SG, independent of any vascular structure, returned to its original shape once deployed.

From the beginning of the spontaneous SG deployment until the end of the endovascular tool removal, a welded contact was activated between the uncovered stent and vascular wall, to represent the hooks that entered the vascular wall during the endovascular procedure. A frictionless contact was imposed between the arterial wall and covered part of the SG.

Challenges associated with the SG deployment, and endovascular tools removal were resolved numerically using the LS-dyna 16 explicit software (Ansys, Canonsburg, Pennsylvania, USA).

\subsection{Results analysis}

During an endovascular procedure, catheterisation of the visceral arteries can become complex if all SG fenestrations are not in front of the ostia. To analyse the adequacy between the fenestrated SG and aorta and anticipate possible catheterisation difficulties, a corrected plan for the fenestrated SG was deduced from the simulation results. The centre of the fenestrations was assumed to correspond to the ostia centre to facilitate catheterisation.

A reference frame $(\vec{n}, \vec{b}, \vec{t})$ was defined on the deformed SG centreline $C L_{S G_{-} \text {def, }}$ as extracted from the graft surface obtained from the simulation results at the time of interest $t$ using VMTK. The centre of each fenestration was set according to the curvilinear abscissa $\alpha(\mathrm{t})$ and angle $\theta(\mathrm{t})$. The binormal vector $\vec{b}$ was collinear to the anteroposterior patient axis, as in clinical practise. The position of the centre of the celiac trunk fenestration was considered as the reference. The relative coordinates of the other fenestrations $(\delta \alpha(t), \delta \theta(t))$ were expressed in terms of this reference, and evaluated at each time $t$. The numerically corrected plan was deduced assuming that the position of the centre of the celiac trunk fenestration corresponded to that of the standard plan.

The difference between the standard plan determined from the preoperative CT scan and the numerically corrected plan can be quantified as follows:

$$
\left\{\begin{array}{l}
\Delta \alpha(t)=\delta \alpha(t)-\delta \alpha_{S P} \\
\Delta \theta(t)=\delta \theta(t)-\delta \theta_{S P}
\end{array}\right.
$$


where $\left(\delta \alpha_{S P}, \delta \theta_{S P}\right)$ represent the relative coordinates extracted from the standard plan.

To quantify the spontaneous SG deployment, the deployment rate was defined as the ratio of the average radius of the compressed SG to its initial radius $R_{0}(0)$. The average radius was calculated from the stent nodes.

\section{Results}

Following the description of the clinical and simulation data, the influences of the main algorithm parameters are analysed in the following subsections, to determine the optimal simulation configuration. The results obtained in a real case are presented in detail. The main steps in simulating the SG deployment are illustrated, including the insertion and removal of the endovascular tools. The adequacy between the SG and vascular structure is then studied, to determine the numerically corrected plan for the fenestrated SG.

\subsection{Clinical data}

Five patients had an SG with four fenestrations, and two had SGs with three fenestrations. Out of the 26 fenestrations, 25 (96\%) were successfully cannulated and stented with covered stents. For one patient (patient 1), the last fenestration (celiac trunk) was impossible to cannulate, as the ostium of the artery and fenestration were not aligned. For all patients, there was no endoleak in the final angiogram, and all aneurisms were excluded. The median time for the operation was $210 \mathrm{~min}$ ( $\min 180$, max $390 \mathrm{~min}$ ), with 65 min of fluoroscopy ( $\min 33, \max 190 \mathrm{~min}$ ). No details regarding the time of each fenestration cannulation were available. For patient 1 , the deployment of the fenestrated SG, bifurcated SG, and limbs required two interventions. The operating and fluoroscopy times for each procedure were accumulated. The median volume of the contrast load was $120 \mathrm{~mL}$ ( $\min 62$, max $180 \mathrm{~min}$ ). The median values of the radiation parameters were $416.8 \mathrm{~Gy} . \mathrm{cm}^{2}$ for the dose area product, and $3744 \mathrm{~Gy}$ for the air kerma. There were no postoperative complications before discharge. On a postoperative CT scan, one type II endoleak was diagnosed; the patient underwent an open conservative conversion 24 months after the initial procedure (aneurysmal sac growth). The patient with the unconnected celiac trunk fenestration occluded the artery without digestive symptoms during follow-up, owing to collaterality. For each patient, the FE simulation was performed several months after the procedure.

\subsection{Simulation data}

First, experiments were conducted on a real case (patient 1) to analyse the influences of the numerical parameters and configure the simulation workflow; only the fenestrated SG was deployed during the endovascular procedure. The bifurcated SG and limbs were deployed during a second endovascular procedure. A postoperative $\mathrm{CT}$ scan was performed seven days after the first procedure to ensure that there were no endoleaks, and to check the SG. This provided a 3D observation of the deployed fenestrated SG. The aorto bi-iliac structure geometry was extracted from the preoperative CT scan. The SG characteristics $\left(\mathrm{N}_{\mathrm{p}}, \mathrm{H}_{\mathrm{G}}, \mathrm{H}_{\mathrm{si}}\right)$ and dimensions are given by the standard plan, as illustrated in Figure $2 \mathrm{a}$.

The algorithm parameters determined in this real case were then used to simulate the SG deployment process in six other patient-specific geometries presenting different iliac tortuosities and calcification rates. These patients were treated using the same type of SG; however, the SG dimensions (diameter and length) were adapted in the simulation according to the standard plan. 
To estimate the coordinates of the release point $P_{\text {deploy }}$ (observable in the reference frame of the postoperative CT scan), it was transposed into the deformed and simulated configurations of the artery just prior to SG deployment. To implement the above, the ostium of the left renal artery was selected as the reference and was assumed to be confused with the centre of the corresponding fenestration, as this artery was catheterised first. This ostium was projected onto the deformed aorta centreline $\mathrm{CL}_{v s_{\text {_def }}}$ as extracted from the vascular structure deformed by the delivery system insertion, to obtain the point $P_{\text {renal }}$. The deployment was then positioned on $\mathrm{CL}_{v \_}$def, such that the curvilinear distance between $\mathrm{P}_{\text {renal }}$ and $\mathrm{P}_{\text {deploy }}$ was equal to the distance written on the SG standard plan (Figure 2a).

\subsection{Algorithm configuration}

\subsubsection{Influence of the compression factor}

The compressed form of the SG, which enables spontaneous deployment, depends on the compression factor $C$. Indeed, the lower the compression factor, the more the SG is compressed in the aorta. To study the influence of this factor on SG deployment, the compression and deployment of the SG were simulated independently of any vascular structure. The evolution of the deployment rate is illustrated in Figure 4, where three compression factors $(C=0.5,0.7$, and 0.9 ) were considered. Regardless of the compression factor, the deployment rate gradually increased until it reached a constant value.

The deployment rate was assumed to converge when the slope of the curve was less than $1 \%$. A constant value of one was obtained when $C=0.7$ and 0.9 . The stents had returned to their original diameter. When $C=0.5$, the deployment rate was slightly lower, and tended to 0.95 .

The duration of the SG deployment corresponded to the time interval $\Delta t$ between the removal of the constraints on the stent node positions and rate of convergence of the deployment. These results show that with increasing compression of the $\mathrm{SG}$, a longer time interval $\Delta t$ is necessary for the SG to return to its original configuration. Thus, the compression factor had a significant influence on the convergence time.

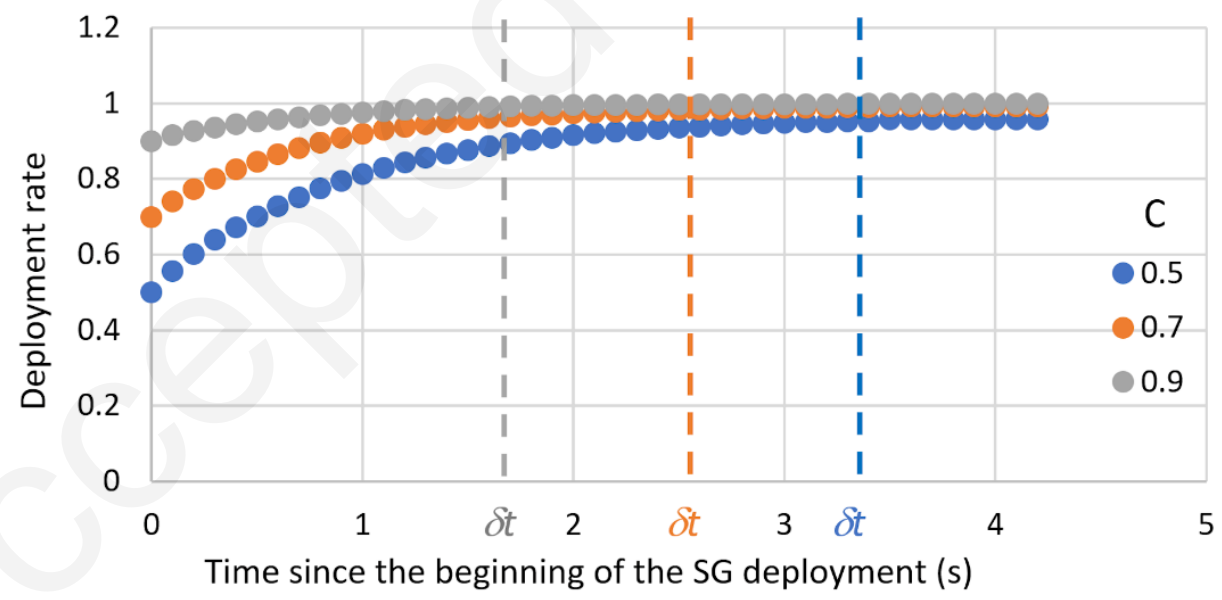

Figure 4: Temporal evolution of the deployment rate of a compressed SG, as deployed independent of any vascular structure, for three compression factors $C$. The vertical bars represent the time from which the deployment rate converged with respect to each of the evaluated compression factors.

A compression factor of $C=0.7$ therefore represents a good compromise between the deployment time and the avoidance of folds outside the vascular structure following the compression of the SG. Owing to preoperative oversizing, the radial size of a SG is typically reduced by $80 \%$ once implanted into a vascular structure. A corrected compression factor $\mathrm{C}^{*}=0.56$ (from $\mathrm{C}=0.7$ ) was applied to account for this oversizing. 


\subsubsection{Influence of the endovascular tool removal}

As no contact was modelled between the tools and SG, removing the endovascular tools at the same time as the SG deployment could be considered. Nevertheless, as the removal of the tools has an influence on the vascular geometry, removing the tools once the deployed SG had reached an equilibrium position in the vascular structure could also be considered, similar to that observed during clinical procedures. To test the above hypotheses, two different time intervals $\Delta t(0.5 \mathrm{~s}$ and $5 \mathrm{~s})$ between the start of the $\mathrm{SG}$ deployment and the start of the tool removal were evaluated.

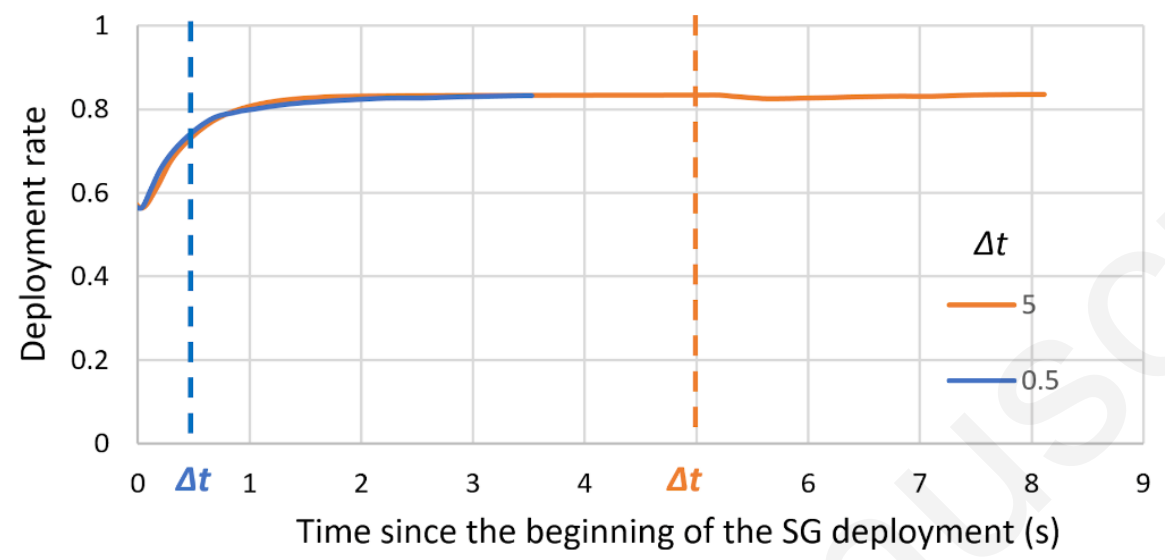

Figure 5: Temporal evolution of the deployment rate of a SG deployed in a vascular structure, with the endovascular tool removal (shown using the vertical bars) starting $0.5 \mathrm{~s}$ or $5 \mathrm{~s}$ after the start of the SG deployment $\left(C^{*}=0.56\right)$.

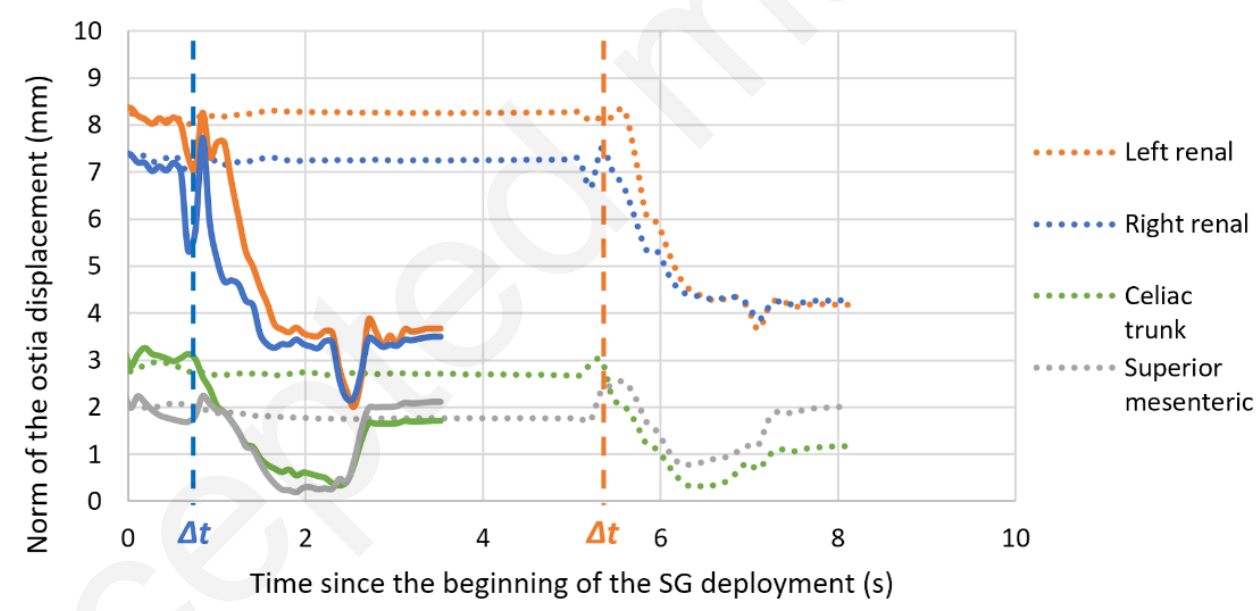

Figure 6: Temporal evolution of the norm of the displacement of the visceral arteries ostia from the beginning of the SG deployment corresponding to $\Delta t=0.5 \mathrm{~s}$ (filled lines) and $\Delta t=5 \mathrm{~s}$ (dotted lines). The vertical bars indicate the endovascular tool removal.

Figure 5 illustrates the temporal evolution of the deployment rate with respect to the two time intervals $\Delta \mathrm{t}$. Regardless of $\Delta \mathrm{t}$, the two curves overlap, and tend toward a maximum deployment rate of 0.83 . Owing to the oversizing, the SG did not reach its nominal diameter and exerted a stress on the vascular wall, thereby preventing endoleaks. The timing of the endovascular tool removal did not affect the maximum deployment rate.

None of the tools straightened the vascular structure when removed. The vascular structure tended to recover its initial configuration, as indicated by the decrease in the displacement of the visceral arteries ostia following tool removal (Figure 6). However, the displacement of the residual ostia remained at the simulation end. The deployed SG induced a residual deformation of the vascular structure following 
removal of the tools. The difference between the residual displacements obtained with $\Delta t=5 \mathrm{~s}$ and $\Delta t=$ $0.5 \mathrm{~s}$, averaged on the four ostia of interest, equalled $0.5 \mathrm{~mm}$. The removal of the tools immediately after the start of the SG deployment slightly altered the final equilibrium between the vascular structure and deployed SG. When the tools were removed at $\Delta t=0.5 \mathrm{~s}$, the aorta and deployed SG did not attain their equilibrium configuration, as opposed to at $\Delta \mathrm{t}=5 \mathrm{~s}$. In this configuration, the ostia displacement stabilisation was observed after $2 \mathrm{~s}$. Thus, a minimum time interval of $2 \mathrm{~s}$ between the start of SG deployment and endovascular tool removal should be set to achieve a correct deployment of the SG.

\subsubsection{Quasi-static hypothesis}

The velocities imposed to compress the SG and insert and remove the endovascular tools were relatively high. These were set up to avoid the occurrence of dynamic phenomena, while maintaining reasonable computational costs. The dynamic effects generated by the SG compression, tool insertion, and removal were negligible, as the inertia of the SG and tools was relatively low compared to that of the vascular structure. Therefore, a quasi-static problem needed to be solved.

However, this problem was resolved using explicit dynamic computation. The ratio between the kinetic energy and total energy from the beginning of the SG compression for the previously defined parameters $\left(C^{*}=0.56\right.$ and $\left.\Delta t=5 s\right)$ is shown in Figure 7 . This ratio was less than $5 \%$ and $1 \%$ during SG compression and deployment, respectively. It increased to $3 \%$ on average when the delivery system was inserted into the left iliac artery and the two guidewires were removed, before decreasing to $0.4 \%$ on average once all tools were removed. The kinetic energy was negligible compared to the total energy, irrespective of the simulation steps of the SG deployment and removal of endovascular tools. Thus, the solved problem constituted a quasi-static problem.

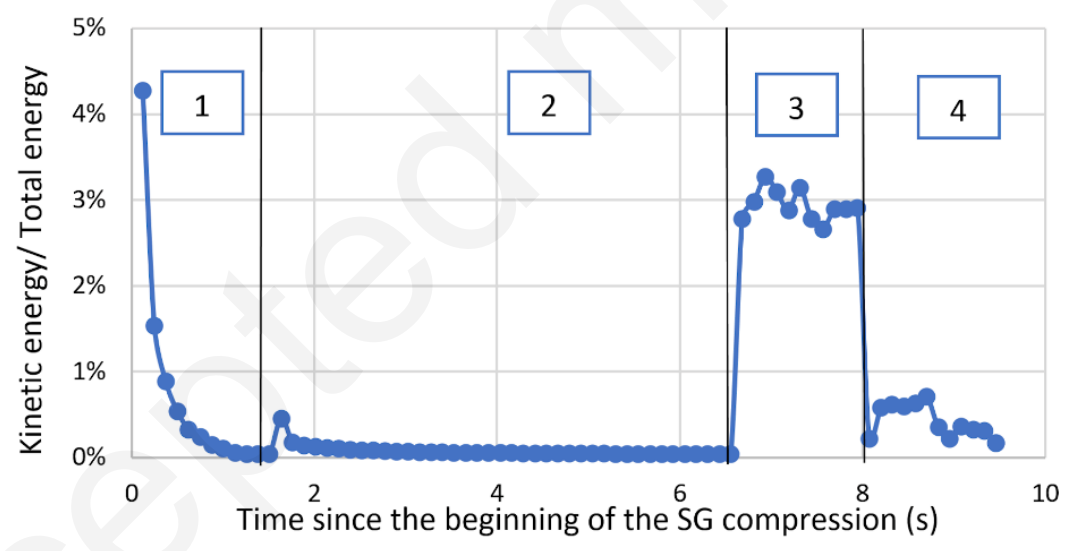

Figure 7: Temporal evolution of the ratio of the kinetic energy to total energy during the simulation steps: (1) SG compression, (2) SG deployment, (3) Removal of the delivery system from the left iliac artery and the stiff guidewires, and (4) Removal of the catheters.

\subsection{Adequacy between the SG and vascular structure}

The adequacy between the vascular structure and SG in patient 1 is analysed in this section.

\subsubsection{SG simulated in a deformed vascular structure}

Figure 8 illustrates the simulation results of the SG deployment process based on the patient data, considering the previously defined parameters. The time required to simulate the insertion of the tools, SG 
deployment, and tool removal with $\Delta t=5 \mathrm{~s}$ was approximately $60 \mathrm{~h}$ using sixteen cores. Notably, the simulation time could be reduced to $42 \mathrm{~h}$ with $\Delta t=2 \mathrm{~s}$.

The SG deployment process was simulated successfully for the six other patient-specific geometries. The preoperative vascular structure, structure deformed by the insertion of the endovascular tools, and final configuration following SG deployment and endovascular tool removal are illustrated for each case in Appendix $C$. Considering the parameters previously defined $\left(C^{*}=0.56\right.$ and $\left.\Delta t=5 \mathrm{~s}\right)$, the simulation required $40 \mathrm{~h}$ on average.

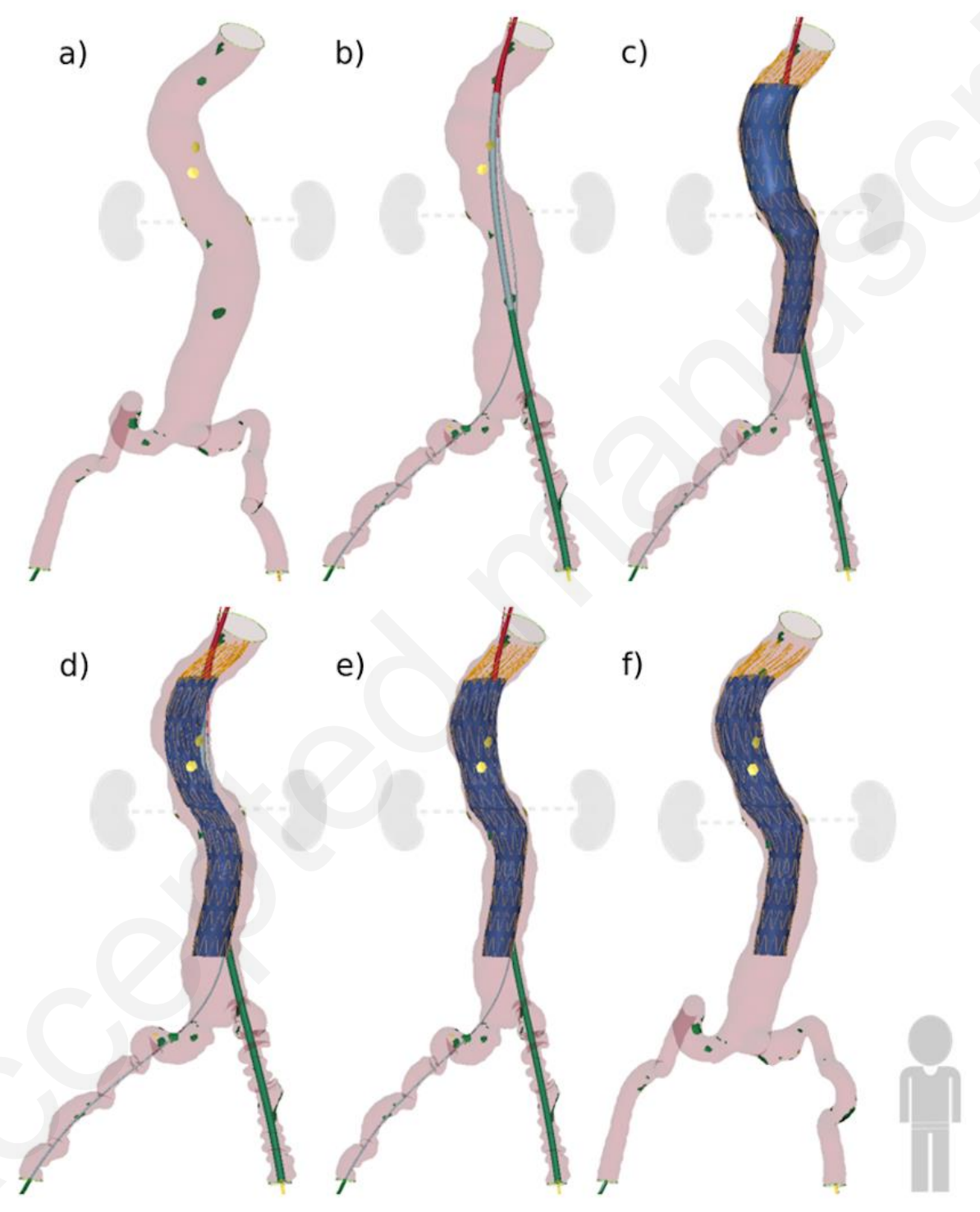

Figure 8: Deformation of the vascular structure seen in patient 1 during the simulation of the SG deployment procedure: a) Vascular structure under blood pressure, b) Insertion of a stiff guidewire in each iliac artery and delivery system in the left iliac artery, c) SG aligned with the deformed centreline, d) SG compressed before deployment, e) Deployed SG, and f) Vascular structure after endovascular tool removal and SG deployment. The collateral arteries ostia are represented in yellow, healthy part of the vascular structure in light red, and calcified part in green. The kidneys are shown as an indication. 
The simulation was performed in the pre-operative reference frame, as opposed to the post-operative frame. A rigid manual pelvis-based registration was performed to compare the simulation results with the postoperative observations recorded on patient 1. Figure 9 a shows the bone segmentations extracted from the pre- and post-operative CT scans in the preoperative reference frame. The overlap of the pelvises was accurate, even when breathing caused a slight shift in the costal bones. The same registration was applied to the stents of the implanted SG segmented on the postoperative CT scans, to represent them in the preoperative reference frame (Figure 9b). The deformed configuration of the graft of the simulated SG after endovascular tool removal was also superimposed onto the preoperative aortoiliac structure in this figure. The simulated and implanted SGs were deployed from the same point. The simulation allowed us to estimate the global form of the implanted SG. A very good adequacy (i.e. alignment) was observed between the implanted SG, simulated SG, and aortoiliac structure at the level of the visceral arteries.

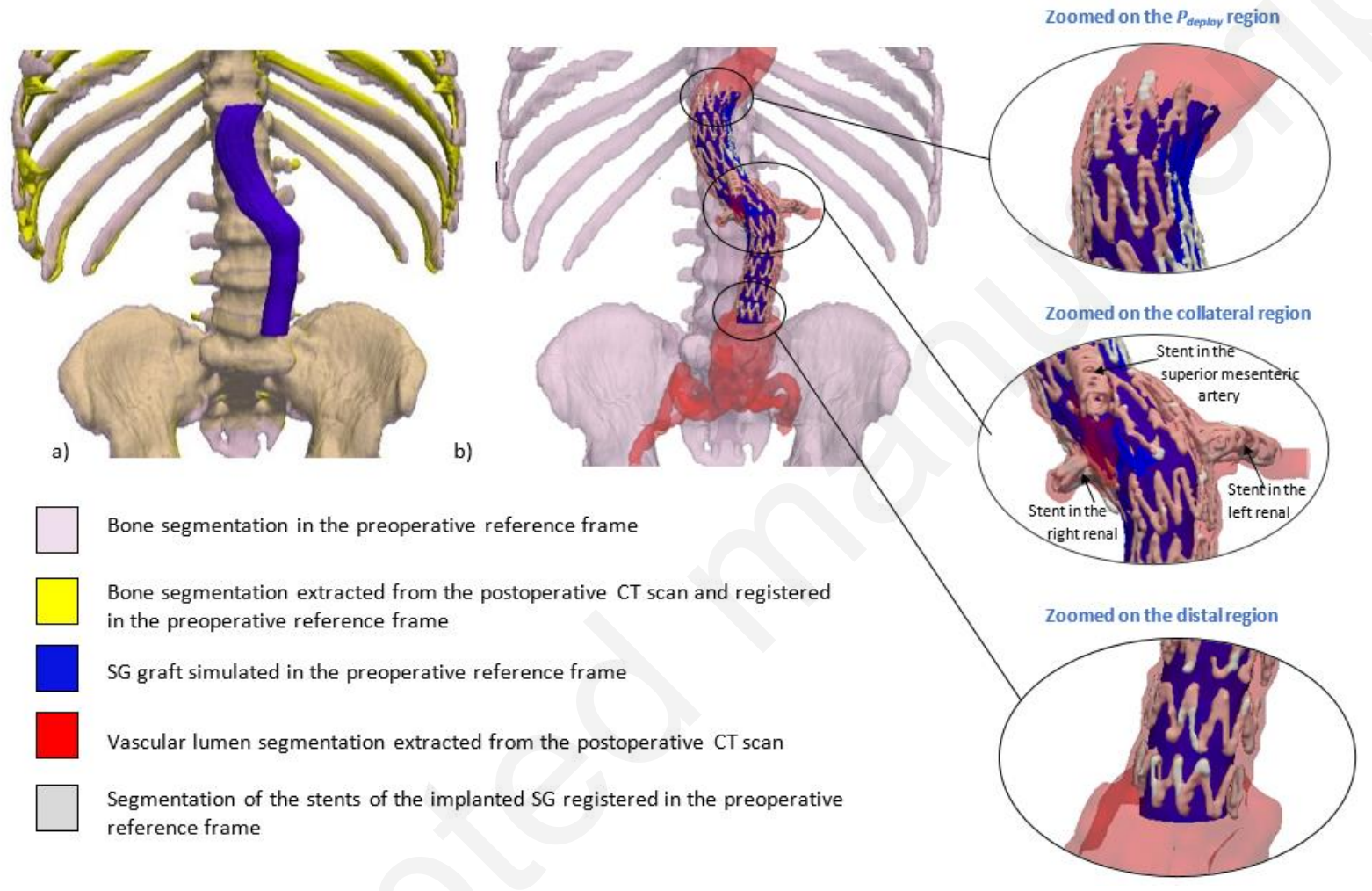

Figure 9: Patient 1: a) Representation of the bone segmentation extracted from the pre-and post-operative CT scans, and the simulated graft in the pre-operative reference frame. b) Representation of the graft of the simulated SG and stents of the implanted SG in the pre-operative aortoiliac structure.

\subsubsection{Determination of the numerically corrected plan}

The insertion of a stiff guidewire in each iliac artery and the delivery system in the left iliac artery induced a displacement of the visceral arteries ostia, as shown in Figure 10. The vascular deformation induced by the insertion of the endovascular tools into the aortoiliac structure was thus considered in the proposed method.

The average displacement of the four visceral ostia was calculated for patient 1 following the insertion of the stiff guidewires and delivery system, SG deployment, and removal of the endovascular tools (Table 1). The insertion of the stiff guidewires caused a slight straightening of the vascular segment, including the ostia of interest. The insertion of the delivery system accentuated the ostia displacement, in particular for the left and right renal arteries, which shifted by $9.74 \mathrm{~mm}$ and $8.52 \mathrm{~mm}$, respectively. 
The most significant displacements were observed for the renal arteries when a stiff guidewire and delivery system were inserted only into the left iliac artery (Table 1 ). The displacement of the right renal artery was larger than previously observed, as no guidewire was inserted in this artery to offset the straightening imposed by the insertion of the tools in the left artery.

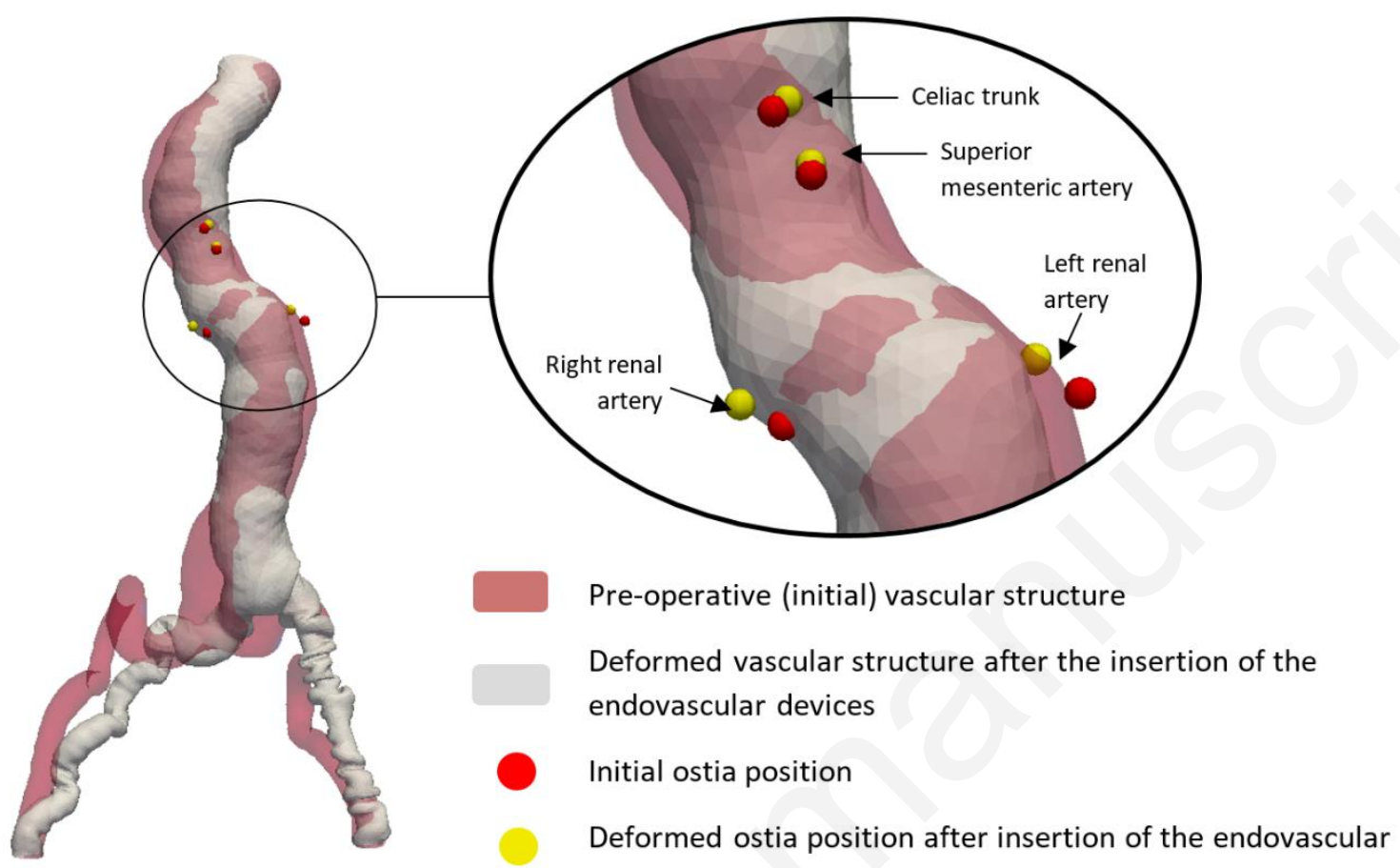

Figure 10: Ostia displacement induced by the insertion of a stiff guidewire in each iliac artery and the delivery system in the left iliac artery.

Regardless of whether one or two guidewires were inserted, the displacement of the renal artery observed after the insertion of the delivery system was greater than $8 \mathrm{~mm}$, i.e. the maximum fenestration diameter. With respect to the six other evaluated vascular geometries, a larger displacement was measured on the celiac trunk and superior mesenteric artery. A mean displacement of $5.4 \mathrm{~mm}$ was obtained on the four ostia of interest. This displacement reached $8.8 \mathrm{~mm}$ following the insertion of the endovascular tools. Therefore, the displacement of the visceral ostia was not negligible, and could add new information regarding the procedure's complexity. The developed method enabled an estimation of the deformed configuration of the SG while considering the deformation induced by the insertion of the tools. 


\begin{tabular}{|c|c|c|c|c|}
\hline & $\begin{array}{l}\text { Left renal } \\
\text { artery }\end{array}$ & $\begin{array}{l}\text { Right renal } \\
\text { artery }\end{array}$ & Celiac trunk & $\begin{array}{c}\text { Superior } \\
\text { mesenteric } \\
\text { artery }\end{array}$ \\
\hline & \multicolumn{4}{|c|}{1 stiff guidewire inserted in each iliac artery } \\
\hline $\begin{array}{l}\text { After insertion of the stiff } \\
\text { guidewires }\end{array}$ & 4.76 & 2.56 & 0.66 & 0.95 \\
\hline $\begin{array}{c}\text { After insertion of the } \\
\text { delivery system }\end{array}$ & 9.74 & 8.52 & 2.99 & 3.17 \\
\hline $\begin{array}{l}\text { After SG deployment and } \\
\text { removal of the tools }\end{array}$ & 4.18 & 4.27 & 1.17 & 1.99 \\
\hline & \multicolumn{4}{|c|}{1 stiff guidewire inserted in the left iliac artery } \\
\hline $\begin{array}{l}\text { After insertion of the stiff } \\
\text { guidewires }\end{array}$ & 4.52 & 4.66 & 1.11 & 1.50 \\
\hline $\begin{array}{c}\text { After insertion of the } \\
\text { delivery system }\end{array}$ & 9.51 & 9.24 & 1.92 & 2.71 \\
\hline $\begin{array}{l}\text { After SG deployment and } \\
\text { removal of the tools }\end{array}$ & 3.57 & 4.13 & 1.62 & 2.17 \\
\hline
\end{tabular}

Table 1: Norm (in $\mathrm{mm}$ ) of the displacement of the four visceral ostia following the insertion of the tools, SG deployment, and removal of the tools for patient 1

In this study, a residual ostia displacement persisted. The aorta did not recover its preoperative configuration following the spontaneous deployment of the SG and removal of the endovascular tools, regardless of the patient anatomy and number of guidewires inserted. In the case of patient 1, a more important displacement was observed with respect to the renal arteries. This displacement was approximately $4.2 \mathrm{~mm}$ when two guidewires were inserted in the left iliac artery, and $4 \mathrm{~mm}$ when only one guidewire was inserted. 


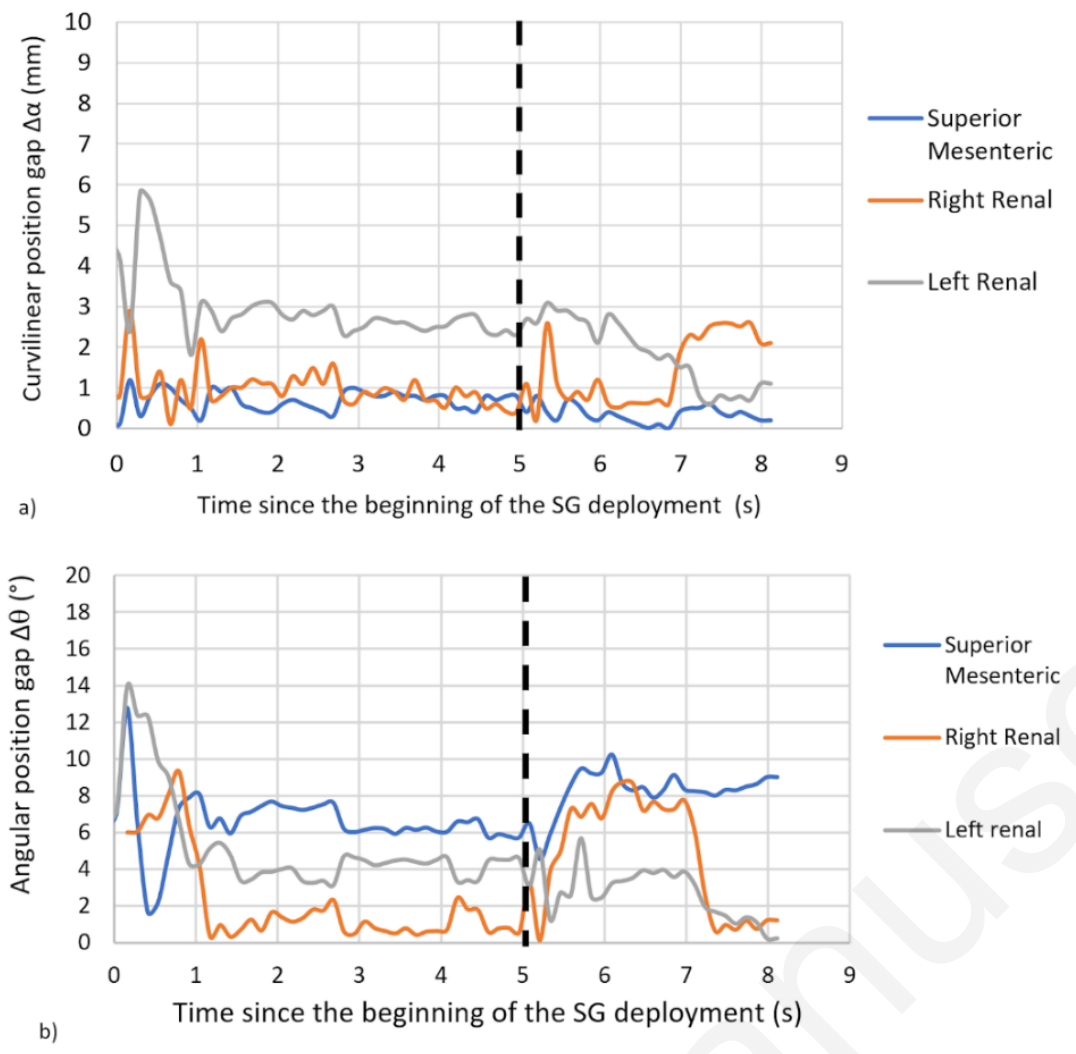

Figure 11: Temporal evolution a) of the curvilinear position gap $\Delta \alpha(t)$ and b) angular position gap $\Delta \theta(t)$ between the centre of each fenestration estimated numerically and that on the standard plan determined from the pre-operative sizing. The dotted line indicates the beginning of the endovascular tool removal.

In the case of patient 1 , the temporal evolutions of the curvilinear $\Delta \alpha(t)$ and angular $\Delta \theta(t)$ position gaps of the superior mesenteric artery and left and right renal arteries in relation to the centre of the celiac trunk fenestration are illustrated in Figure 11. Strong oscillations in $\Delta \alpha(t)$ and $\Delta \theta(t)$ were observed during the SG deployment. The curvilinear and angular position gaps reached their maximum values $\left(5.6 \mathrm{~mm}\right.$ and $\left.14^{\circ}\right)$ for the left renal artery. As the radius of an 8-mm fenestration corresponded to an angle of $12.7^{\circ}$, the curvilinear and angular position gaps of the left renal artery were greater than the limit of fenestration. The fenestration of the left renal artery as predicted on the corrected plan did not overlap with that of the standard plan. These maximum values were attained when the SG was $2 / 3$ deployed, i.e. when the ostia were catheterised during the endovascular procedure. The curvilinear and angular position gaps obtained for the superior mesenteric artery and right renal artery were below the limits, but were not zero. The fenestrations of these two ostia overlapped partially. 


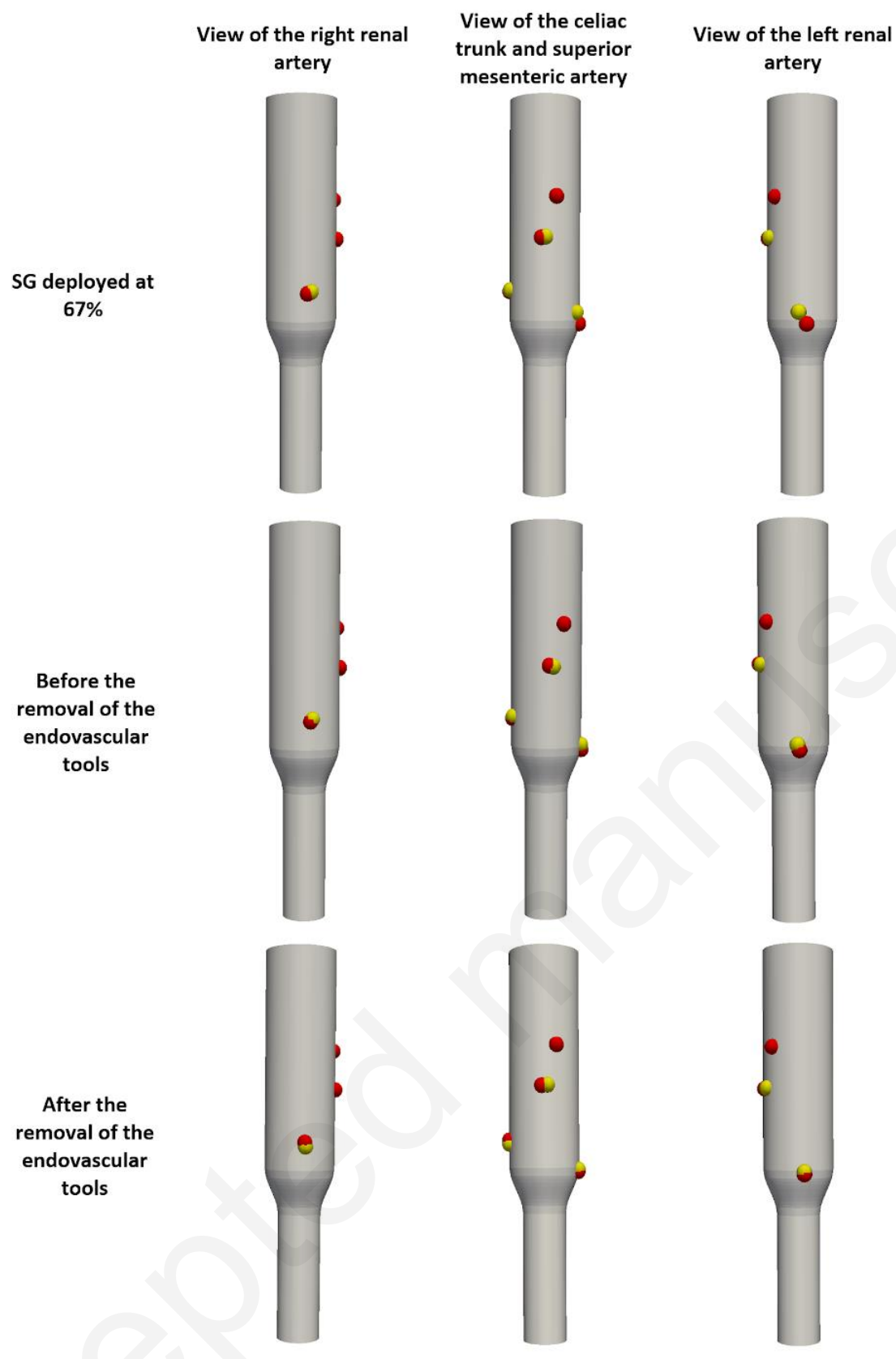

Figure 12: Comparison between the standard plan (in red) and numerically corrected plan (in yellow). The fenestrations are represented by spheres, each with a radius of $8 \mathrm{~mm}$.

When the SG reached its maximum deployment rate following the insertion of the endovascular tools at $t$ $=2 \mathrm{~s}$, the curvilinear and angular position gap oscillated slightly around an average value that was inferior to the fenestration limits (Figure 11). When the SG reached its equilibrium position before the removal of the endovascular tools, the fenestrations of the corrected plan overlapped partially with those of the standard plan. The removal of the tools induced variations in $\Delta \alpha(\mathrm{t})$ and $\Delta \theta(\mathrm{t})$, which also remained below the fenestration limits. Following the removal of the endovascular tools, the fenestrations of the numerically corrected plan also overlapped partially with those of the standard plan.

The numerically corrected plan as determined at three time points of interest (when the SG was deployed at $67 \%$ and before and after the removal of the endovascular tools) was superimposed onto the standard 
plan (Figure 12). Comparisons between the two plans confirmed that when the SG was deployed at $67 \%$, the fenestrations of the left renal artery did not overlap at all, whereas those of the superior mesenteric and right renal arteries overlapped partially. Before and after the removal of the endovascular tools, differences persisted between the standard and numerically corrected plans, and partial overlapping among all of the fenestrations was observed.

To complete the results, the correlation between the simulation and intraoperative data was analysed. For the seven cases and each target vessel, the mean value of the displacement of the ostium was correlated with the intraoperative data (contrast load, time of fluoroscopy). The correlation was studied two times: after endovascular tool insertion, and after SG deployment and endovascular tool removal. The results of the correlations are reported in Tables 2 and 3. It appears that the displacement of the left renal ostium is strongly correlated with the fluoroscopy time.

\begin{tabular}{|c|c|c|c|c|c|c|}
\hline & $\begin{array}{c}\mathrm{M} 1^{*} \\
(\mathrm{~mm})\end{array}$ & Correlation $\mathrm{Cl} 95 \%^{* *}$ & $\mathrm{p}$-value & $\begin{array}{c}\mathrm{M} 2^{*} \\
(\mathrm{~mm})\end{array}$ & Correlation $\mathrm{Cl} 95 \%^{* *}$ & $\begin{array}{c}\mathrm{p}- \\
\text { value }\end{array}$ \\
\hline $\begin{array}{c}\text { Right renal } \\
\text { artery }\end{array}$ & 6.14 & $0.286[-0.5955 ; 0.8548]$ & 0.53 & 1.9 & $0.613[-0.2598 ; 0.9347]$ & 0.14 \\
\hline $\begin{array}{c}\text { Left renal } \\
\text { artery }\end{array}$ & 6.14 & $0.832[0.2117 ; 0.9745]$ & 0.02 & 2 & $0.622[-0.246 ; 0.9365]$ & 0.13 \\
\hline $\begin{array}{c}\text { Superior } \\
\text { mesenteric } \\
\text { artery }\end{array}$ & 5.18 & $-0.538[-0.9188 ; 0.3618]$ & 0.21 & 1.9 & $-0.162[-0.8157 ; 0.673]$ & 0.73 \\
\hline Celiac trunk & 4.59 & $-0.503[-0.911 ; 0.4027]$ & 0.25 & 1.8 & $-0.414[-0.8897 ; 0.4925]$ & 0.36 \\
\hline
\end{tabular}

* M1, mean displacement (in $\mathrm{mm}$ ) resulting from the simulation of the endovascular tools insertion, and * M2, mean displacement (in $\mathrm{mm}$ ) resulting from the simulation of the SG deployment and endovascular tools removal, ** $95 \%$ confidence interval

Table 2: Pearson correlation coefficient between the fluoroscopy duration and norm of the displacement of the four visceral ostia

\begin{tabular}{|c|c|c|c|c|c|c|}
\hline & $\mathrm{M} 1^{*}$ & Correlation $\mathrm{Cl} 95 \% * *$ & $\mathrm{p}$-value & $\mathrm{M} 2^{*}$ & Correlation $\mathrm{Cl}$ ** & $\mathrm{p}$-value \\
\hline $\begin{array}{c}\text { Right renal } \\
\text { artery }\end{array}$ & 6.14 & $0.613[-0.2598 ; 0.9347]$ & 0.14 & 1.9 & $0.048[-0.7316 ; 0.773]$ & 0.92 \\
\hline $\begin{array}{c}\text { Left renal } \\
\text { artery }\end{array}$ & 6.14 & $0.622[-0.246 ; 0.9365]$ & 0.13 & 2 & $0.533[-0.3669 ; 0.9178]$ & 0.22 \\
\hline $\begin{array}{c}\text { Superior } \\
\text { mesenteric } \\
\text { artery }\end{array}$ & 5.18 & $-0.162[-0.8157 ; 0.673]$ & 0.73 & 1.9 & $-0.131[-0.8048 ; 0.6899]$ & 0.78 \\
\hline Celiac trunk & 4.59 & $-0.414[-0.8897 ; 0.4925]$ & 0.36 & 1.8 & $-0.52[-0.9149 ; 0.3826]$ & 0.23 \\
\hline
\end{tabular}

*M1, mean displacement (in $\mathrm{mm}$ ) resulting from the simulation of the endovascular tools insertion, and * $\mathrm{M} 2$, mean displacement (in $\mathrm{mm}$ ) resulting from the simulation of the SG deployment and endovascular tools removal, ** $95 \%$ confidence interval

Table 3: Pearson correlation coefficients between the contrast load and norm of the displacement of the four visceral ostia 


\section{Discussion}

As endovascular procedures are being used to treat increasingly complex diseases, the development of efficient planning tools is necessary to ensure a successful procedure. In the case of FEVAR procedures, the risks of complications are not negligible, and the expectation of adequacy between the SG and visceral vascular segment is important. This study proposed a finite element method for simulating the FEVAR procedure. The problems associated with the insertion of the endovascular tools, SG deployment, and removal of the tools in a deformable vascular structure were solved using an explicit method.

Motivated by the development of endovascular treatments in recent years, initial studies focused on the deployment of an uncovered stent in generic geometries [30]-[33] or in patient-specific geometries of the carotid artery [33], [34], and peripheral arteries [36], [37]. However, none of these studies considered the insertion of endovascular tools before simulating the deployment phase, which can have a significant influence, particularly in the case of FEVAR.

In FEVAR simulations, considering an SG is an additional difficulty. Indeed, the deployment of an SG in a vessel is a non-linear problem, owing to the formation of graft folds, use of non-linear materials, and involvement of complex contacts between the SG and the vascular wall. Solving such a problem remains difficult, even today. The few studies [14], [15], [17], [20] that have addressed the problem have focussed on the deployment phase, without considering the vascular deformation induced by the insertion of endovascular tools. Only [21] recently proposed simulating the entire deployment process of a SG while considering the vascular deformation. After finding the mechanical equilibrium between the stiff guidewire and vascular structure, the SG was compressed in the sheath of the delivery system, and was progressively introduced into the iliac artery until its target position. The sheath was then removed to release the SG into the vascular structure. Even if a realistic method is proposed for unsheathing the SG, this study does not address the issue of adapting the SG plan to the anatomy. This interesting work focused on intraoperative SG rotation, and some simplifying assumptions were made regarding the presence of calcifications, support of surrounding tissues, and SG material parameters (graft thickness, elastic stents).

The proposed numerical method enabled the main steps of the fenestrated SG deployment procedure to be reproduced, including the insertion and removal of the endovascular tools. The finite element calculations (especially in nonlinear cases) can provide different responses according to the parameter settings. As previously mentioned, the numerical simulation of the vascular deformation was based on the method described in [16], [38], in which the influence of the deformation parameters had already been analysed. In the proposed work, the influences of the main parameters related to SG deployment (compression factor, time of tool removal) were analysed, to thereby show the robustness and reproducibility of the method.

The dilation step performed at the end of the clinical procedure was not simulated. In addition, during the endovascular procedure, the surgeon could perform corrections, orient the devices to adapt to his gestures, and guide the SG deployment. This is difficult to reproduce in silico. Moreover, numerically, the SG was aligned along the aorta centreline, and not along the delivery system. As the latter could be located very close to the vascular wall, this would require a strong compression of the SG, and could cause problems related to graft folding. The alignment with the aorta centrelines could influence the final position of the SG. Nevertheless, this method limited the formation of graft folds [39], [40].

Despite the above-mentioned limitations, the developed finite element method proved to be robust. The SG deployment process was simulated successfully on seven patient-specific geometries presenting 
different anatomical characteristics, i.e. those which could strongly affect the vascular deformation and SG deployment.

In this study, the computation time was another limitation. Considerable time was required to simulate the entire procedure, as many actions and contacts had to be modelled. In comparison, the simulation of the SG deployment in the aneurysmal part of a synthetic aorta developed by Roy et al. [29] took $20 \mathrm{~h}$ on 56 cores, without considering the insertion of the tools. Therefore, the computation time of our simulation is not surprising. The development of reduced-order modelling approaches could provide an interesting approach to addressing this issue.

A 3D comparison between the simulated SG and postoperative images provided satisfactory results (Figure 9). The proposed numerical method enabled an estimation of the residual vascular deformation owing to the SG deployment. When the standard plan was established, an oversizing of $20 \%$ was considered. This oversizing was maintained to model the simulated SG. The SG was deployed at $83 \%$ when it reached its equilibrium position. Its diameter was, at that point, slightly larger than the initial diameter of the aorta. The SG remained compressed and exerted pressure on the arterial wall to prevent endoleaks. Nevertheless, the deployment process induced the deformation of the abdominal aorta and residual displacement of the ostia of interest. The estimation of the fenestration position from the numerical results considered this deformation. The position of the fenestrations on the numerically corrected plan was defined by locating the ostia in the simulated SG reference frame during its deployment, before and after the removal of the endovascular tools. Thus, the SG fenestrations predicted in silico were perfectly located in front of the visceral arteries ostia, facilitating catheterisation. However, the fenestrations of the numerically corrected plan did not coincide with those of the standard plan, which are usually determined from a preoperative CT (particularly during catheterisation). These differences might explain the difficulties encountered during catheterisation, and support the observation that an SG plan could be optimised based on a pre-operative simulation.

To compare the plans, we considered the fenestrations' centre and ostia as fixed points. In practise, once a collateral artery has been catheterised, the surgeon can slightly move the SG to catheterise the other ostia. This procedure is easy to perform initially and enables catheterisation of the second ostium, but becomes increasingly difficult with an increase in the number of catheterised arteries. Problems might also occur when stents are deployed in collateral arteries.

The results obtained from the intraoperative data showed that fluoroscopy time is correlated to the simulated displacement of the left renal ostium at the time when the stiff guidewires and delivery system remain inserted. When the endovascular tools are removed, there is no correlation. This suggests that the delivery system is stiffer than the SG. It also suggests that the anatomy returns to its initial shape after SG deployment. In this case, there should be no risk of postoperative complications, such as stent occlusion. Therefore, the deformations are mainly an intraoperative difficulty. Even if more patients are required to draw conclusions regarding the correlation and therefore the potential clinical interest, the initial results are encouraging.

The fenestrated SGs are custom-made, and cost more than classical SGs. However, it is not uncommon for a surgeon to encounter catheterisation problems related to the improper positioning of the fenestrations during the procedure. The development of numerical simulations for SG deployment that incorporate the vascular deformation induced by the insertion of endovascular tools to determine the corrected plan could help anticipate catheterisation problems, e.g. based on comparisons between the standard and numerically corrected plans. 
This work is the first step towards a clinical study comparing intra and immediate postoperative data between patients with complex anatomies (tortuosity and angulation) and those without. Afterwards, and with strong clinical data, the assistance of a finite element simulation is suggested. Another study could be conducted to compare the same clinical data, but for patients with and without simulated assisted planning. Other brands of SGs can also be modelled. This approach requires help and discussion from manufacturers (Cook, Aortic Terumo, Jotec-Cryolife) to consider the data provided by finite element simulation in the design and planning of fenestrated SGs.

\section{Conclusion}

In this article, a patient-specific approach based on the finite element method was presented, for simulating the procedure of SG deployment in a juxtarenal aneurism aorta. A method was also proposed for determining the corrected plan of a fenestrated SG based on numerical results. The finite element method considered the vascular deformations induced by the insertion of the stiff guidewires and delivery system into the iliac arteries during FEVAR. The effects of the main algorithm parameters were also analysed. It was demonstrated that the results obtained using the proposed numerical approach are similar to those from post-deployment CT observations. The proposed numerical approach demonstrated good performance relative to the post-deployment CT observations. The numerical simulations were performed successfully for seven representative cases to demonstrate the reproducibility and robustness of the proposed method.

The difference in the positioning of the renal artery fenestration on the standard (defined based on the preoperative $\mathrm{CT}$ ) and numerically corrected plans revealed the catheterisation difficulties encountered during endovascular treatment. In future studies, incorporating such simulations during the FEVAR planning step could enable surgeons to anticipate any potential complications.

\section{Acknowledgements}

This work was partially supported by the French National Research Agency (ANR) within the framework of the Investissement d'Avenir Program through Labex CAMI (ANR-11- LABX-0004). 


\section{Appendix A: Summary of the vascular deformation model induced by the insertion of the endovascular tools}

The methods employed to create the vascular geometry, determine the boundary conditions, and insert the endovascular tools (based on the literature published by Gindre et al. [16]) are summarised here.

\section{A. Aortoiliac geometry}

The aorto bi-iliac structure was extracted from a preoperative thoracoabdominal CT scan, using the sizing software Endosize ${ }^{\circledR}$. The aortoiliac geometry represented the vessel lumen only, which could be easily segmented on the $\mathrm{CT}$, and did not represent the collateral arteries, whose elastic support was considered in the boundary conditions (cf. § B). The effect of the intraluminal thrombus was neglected. The arterial wall was meshed with triangular shell elements with thicknesses of $1.5 \mathrm{~mm}$ and $1.2 \mathrm{~mm}$ for the aorta and iliac arteries, respectively.

The calcifications identified on the pre-operative CT were transferred to the mesh size to differentiate the calcified elements from those modelling the healthy wall. The mechanical behaviour of the healthy wall was described based on the hyperelastic, isotropic, non-linear, and polynomial Yeoh law. A linear elastic behaviour was considered to represent the mechanical behaviour of the calcifications (Appendix $B$ ).

\section{B. Modelling the external support}

The aortoiliac structure is surrounded by muscles and bones, which affect its deformation when endovascular tools are inserted. To appropriately model this external environment, a viscoelastic support was applied to each node of the mesh of the vascular structure. The latter was calculated as the sum of three components: (1) the effects of the fluids and soft tissues of the retroperitoneal cavity, (2) the natural damping forces caused by the tissues and biological fluids in contact with the vascular structure, and (3) the elastic support provided by the bones and internal iliac arteries.

\section{Modelling the insertion of the endovascular tools}

After pressurising the 'blood pressure free' geometry, all endovascular tools (catheters, stiff guidewires, and delivery system) were introduced by imposing a velocity at their distal end until they were fully inserted into the vascular structure. A flexible tube-like thin catheter meshed with shell elements was inserted. Stiff guidewires, as meshed with beam elements representing the Lunderquist ${ }^{\circledR}$ (Cook) 'extrastiff' guidewire used in the clinic, were then introduced into the catheter. A delivery system meshed with shell elements (whose mechanical properties varied along its length) for modelling the flexible distal end and part with the compressed SG were introduced up to the deployment site in the iliac artery, as determined during sizing. 
Appendix B: Simulation parameters

\begin{tabular}{|c|c|c|c|}
\hline & Elements type & Parameters & Values \\
\hline \multirow{2}{*}{$\begin{array}{l}\text { Catheter } \\
\text { (Elastic } \\
\text { material) }\end{array}$} & \multirow{2}{*}{ Shell } & Poisson's ratio & 0.3 \\
\hline & & Young's modulus & $500 \mathrm{MPa}$ \\
\hline \multirow{11}{*}{$\begin{array}{l}\text { Stiff guidewires } \\
\text { (Elastic } \\
\text { material) }\end{array}$} & \multirow{11}{*}{ Beam } & \multicolumn{2}{|c|}{ Soft Part (Proximal end) } \\
\hline & & Poisson's ratio & 0.3 \\
\hline & & Young's modulus & $\begin{array}{l}10000 \\
\mathrm{MPa}\end{array}$ \\
\hline & & Length & $40 \mathrm{~mm}$ \\
\hline & & \multicolumn{2}{|c|}{ Medium Part } \\
\hline & & Poisson's ratio & 0.3 \\
\hline & & Young's modulus & $\begin{array}{l}50000 \\
\mathrm{MPa}\end{array}$ \\
\hline & & Length & $80 \mathrm{~mm}$ \\
\hline & & \multicolumn{2}{|c|}{ Stiff Part (Distal end) } \\
\hline & & Poisson's ratio & 0.3 \\
\hline & & Young's modulus & $\begin{array}{l}180000 \\
\mathrm{MPa}\end{array}$ \\
\hline \multirow{12}{*}{$\begin{array}{l}\text { Delivery system } \\
\text { (Elastic } \\
\text { material) } \\
\text { (experimental } \\
\text { data) }\end{array}$} & \multirow{12}{*}{ Shell } & \multicolumn{2}{|c|}{ Tip (Proximal end) } \\
\hline & & Poisson's ratio & 0.45 \\
\hline & & Young's modulus & $100 \mathrm{MPa}$ \\
\hline & & Length & $80 \mathrm{~mm}$ \\
\hline & & \multicolumn{2}{|c|}{ Part representing the compressed SG } \\
\hline & & Poisson's ratio & 0.45 \\
\hline & & Young's modulus & $200 \mathrm{MPa}$ \\
\hline & & Length & $\begin{array}{c}=S G \\
\text { length }\end{array}$ \\
\hline & & Mean radius & $2.475 \mathrm{~mm}$ \\
\hline & & & \\
\hline & & Poisson's ratio & 0.45 \\
\hline & & Young's modulus & $330 \mathrm{MPa}$ \\
\hline
\end{tabular}

Table B.1: Mechanical and geometrical properties of the endovascular tools

\begin{tabular}{|l|l|}
\hline Speed & Values \\
\hline Catheter & $800 \mathrm{~mm} / \mathrm{s}$ \\
\hline Stiff guidewires & $800 \mathrm{~mm} / \mathrm{s}$ \\
\hline Delivery system & $300 \mathrm{~mm} / \mathrm{s}$ \\
\hline
\end{tabular}

Table B.2: Insertion and removal speed of the endovascular tools 


\begin{tabular}{|c|l|l|l|}
\hline & Elements type & Parameters & Values \\
\hline \multirow{3}{*}{$\begin{array}{c}\text { Healthy wall } \\
\text { (hyperelastic } \\
\text { material) }\end{array}$} & \multirow{3}{*}{ Shell } & Poisson's ratio & 0.49 \\
\cline { 3 - 4 } & $\mathrm{C}_{10}$ & $0.005 \mathrm{MPa}$ \\
\cline { 3 - 4 } $\begin{array}{c}\text { Calcified wall } \\
\text { (elastic } \\
\text { material) }\end{array}$ & $\mathrm{C}_{20}$ & $0.02 \mathrm{MPA}$ \\
\cline { 3 - 4 } & $\mathrm{C}_{30}$ & $0 \mathrm{MPa}$ \\
\hline \multirow{2}{*}{ Shell } & Young's modulus & $40 \mathrm{MPa}$ \\
\cline { 3 - 4 } & Poisson's ratio & 0.4 \\
\hline
\end{tabular}

Table B.3: Mechanical properties of the vascular structure [16]

\begin{tabular}{|c|c|c|c|}
\hline & $\begin{array}{c}\text { Elements } \\
\text { type }\end{array}$ & Parameters & Values \\
\hline \multirow{9}{*}{$\begin{array}{c}\text { Nitinol } \\
\text { Stents } \\
{[25],[27]}\end{array}$} & \multirow{9}{*}{ Beam } & Austenite elasticity modulus & $40000 \mathrm{MPa}$ \\
\hline & & Austenite Poisson's ratio & 0.46 \\
\hline & & Martensite elasticity modulus & $18554 \mathrm{MPa}$ \\
\hline & & Martensite Poisson's ratio & 0.46 \\
\hline & & Transformation strain & 0.04 \\
\hline & & Stress at the beginning of transformation (loading) & $390 \mathrm{MPa}$ \\
\hline & & Stress at the end of transformation (loading) & $425 \mathrm{MPa}$ \\
\hline & & Stress at the beginning of transformation (unloading) & $140 \mathrm{MPa}$ \\
\hline & & Stress at the end of transformation (unloading) & $135 \mathrm{MPa}$ \\
\hline \multirow{3}{*}{$\begin{array}{c}\text { Graft made } \\
\text { of PET } \\
\text { (elastic } \\
\text { material } \\
{[26] \text { ) }}\end{array}$} & \multirow[b]{3}{*}{ Shell } & Circumferential Young's modulus & $1000 \mathrm{MPa}$ \\
\hline & & Longitudinal Young's modulus & $225 \mathrm{MPa}$ \\
\hline & & Poisson's ratio & 0.3 \\
\hline
\end{tabular}

Table B.4: Mechanical properties of the stent graft 
Appendix C: Simulation results corresponding to patients 2-7 with different tortuosity and calcification configurations In the following images, the healthy part of the vascular structure is represented in light red, and the calcified part in green. The kidneys are shown as an indication.

Figure C: Vascular structure Patient

Vascular structure extracted from the preoperative $\mathrm{CT}$ scan

Vascular structure deformation

Vascular structure after SG caused by the insertion of the deployment and endovascular endovascular tools

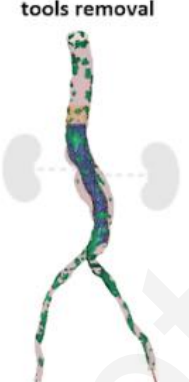
extracted from the pre-operative CT scan, vascular structure deformation following the insertion of the endovascular tools, and that following the SG deployment and endovascular tools removal for patients 2-7.
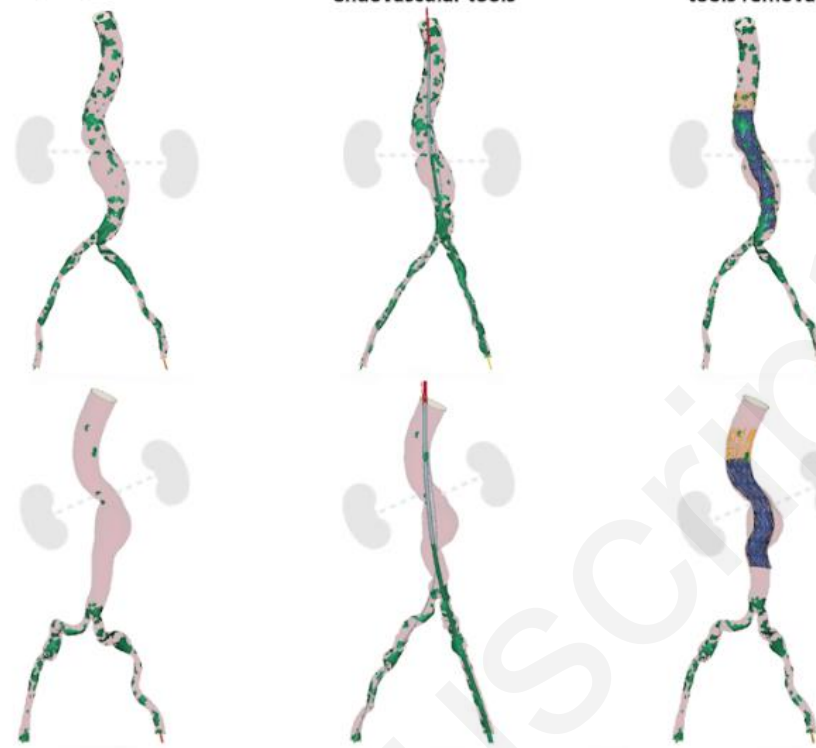

3
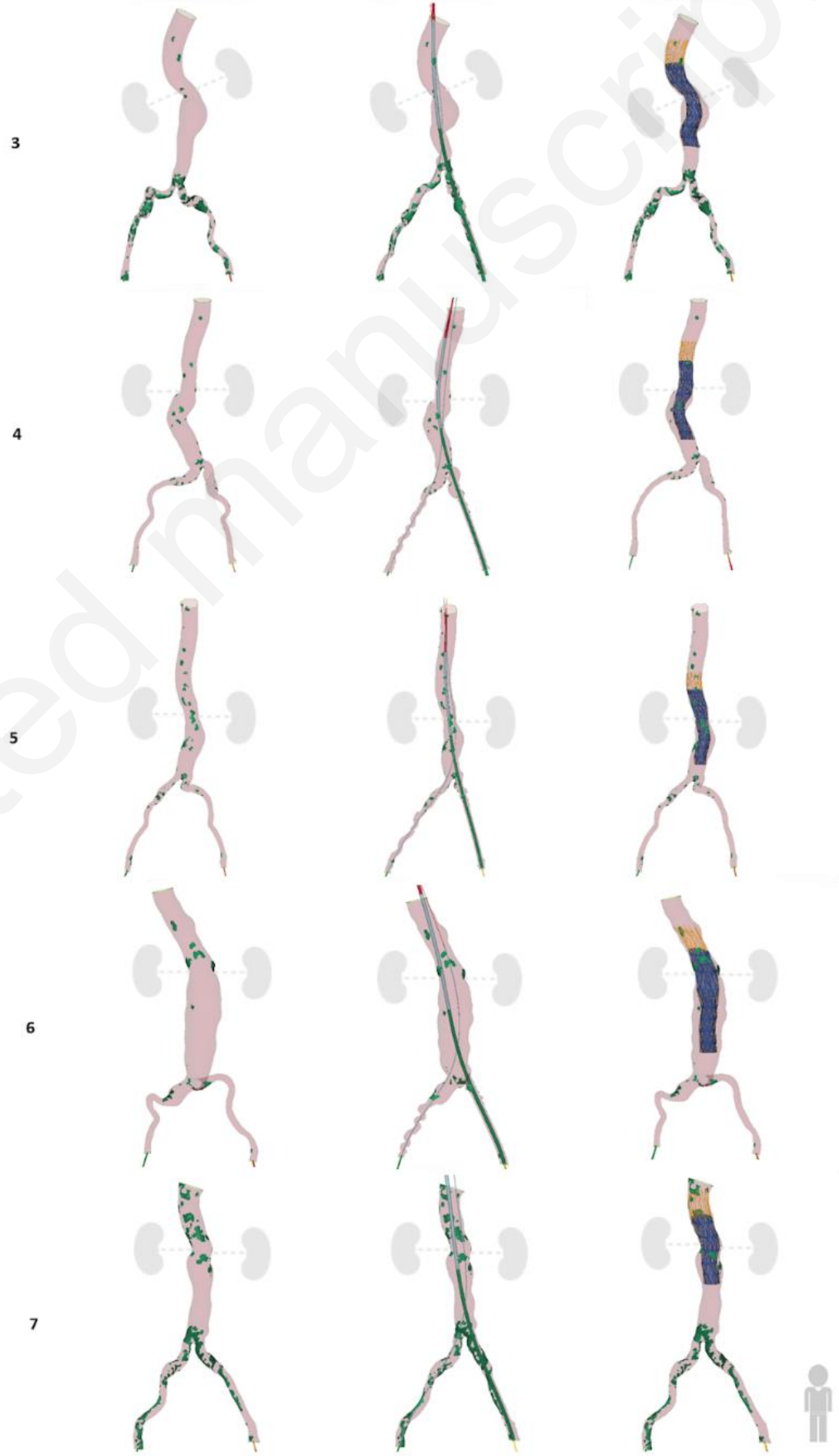


\section{Bibliography}

[1] C. K. Zarins et al., "Will endovascular repair replace open surgery for abdominal aortic aneurysm repair?," Ann. Surg., vol. 232, no. 4, pp. 501-507, 2000, doi: 10.1097/00000658-200010000-00005.

[2] Z. Hu, Y. Li, R. Peng, J. Liu, T. Zhang, and W. Guo, "Experience with fenestrated endovascular repair of juxtarenal abdominal aortic aneurysms at a single center," Med. (United States), vol. 95, no. 10, pp. 1-7, 2016, doi: 10.1097/MD.0000000000002683.

[3] M. J. Eagleton, M. Follansbee, K. Wolski, T. Mastracci, and Y. Kuramochi, "Fenestrated and branched endovascular aneurysm repair outcomes for type II and III thoracoabdominal aortic aneurysms," J. Vasc. Surg., vol. 63, no. 4, pp. 930-942, 2016, doi: 10.1016/j.jvs.2015.10.095.

[4] D. E. Timaran et al., "Gender and perioperative outcomes after fenestrated endovascular repair using custom-made and off-the-shelf devices," J. Vasc. Surg., vol. 64, no. 2, pp. 267-272, 2016, doi: 10.1016/j.jvs.2016.02.062.

[5] K. H. J. Ultee, S. L. Zettervall, P. A. Soden, J. Darling, H. J. M. Verhagen, and M. L. Schermerhorn, "Perioperative outcome of endovascular repair for complex abdominal aortic aneurysms," J. Vasc. Surg., vol. 65, no. 6, pp. 1567-1575, 2017, doi: 10.1016/j.jvs.2016.10.123.

[6] G. S. Oderich et al., "Results of the United States multicenter prospective study evaluating the Zenith fenestrated endovascular graft for treatment of juxtarenal abdominal aortic aneurysms," J. Vasc. Surg., vol. 60, no. 6, pp. 1420-1428.e5, 2014, doi: 10.1016/j.jvs.2014.08.061.

[7] T. M. Mastracci, M. J. Eagleton, Y. Kuramochi, S. Bathurst, and K. Wolski, "Twelve-year results of fenestrated endografts for juxtarenal and group IV thoracoabdominal aneurysms," J. Vasc. Surg., vol. 61, no. 2, pp. 355-364, 2015, doi: 10.1016/j.jvs.2014.09.068.

[8] B. W. Starnes, R. E. Heneghan, and B. Tatum, "Midterm results from a physician-sponsored investigational device exemption clinical trial evaluating physician-modified endovascular grafts for the treatment of juxtarenal aortic aneurysms," J. Vasc. Surg., vol. 65, no. 2, pp. 294-302, 2017, doi: 10.1016/j.jvs.2016.07.123.

[9] B. Maurel et al., "Evaluation of Visceral Artery Displacement by Endograft Delivery System Insertion," J. Endovasc. Ther., vol. 21, no. 2, pp. 339-347, 2014, doi: 10.1583/13-4471MR.1.

[10] K. M. Meess et al., "3D printed abdominal aortic aneurysm phantom for image guided surgical planning with a patient specific fenestrated endovascular graft system," Proc SPIE Int. Soc. Opt. Eng., vol. 10138, p. 101380P, 2017, doi: 10.1117/12.2253902.

[11] R. J. Winder, Z. Sun, B. Kelly, P. K. Ellis, and D. Hirst, "Abdominal aortic aneurysm and stent graft phantom manufactured by medical rapid prototyping," J. Med. Eng. Technol., vol. 26, no. 2, pp. 75-78, 2002, doi: 10.1080/03091900210124404.

[12] F. Taher et al., "The influence of prototype testing in three-dimensional aortic models on fenestrated endograft design," J. Vasc. Surg., vol. 65, no. 6, pp. 1591-1597, 2017, doi: 10.1016/j.jvs.2016.10.108.

[13] B. W. Starnes, B. Tatum, and N. Singh, "Procedural and perioperative results in patients treated with fenestrated endovascular aneurysm repair planned by automated software in a physiciansponsored investigational device exemption trial of physician-modified endografts," J. Vasc. Surg., vol. 68 , no. 5, pp. 1297-1307, 2018, doi: 10.1016/j.jvs.2018.02.045.

[14] S. De Bock et al., "Virtual evaluation of stent graft deployment: A validated modeling and simulation study," J. Mech. Behav. Biomed. Mater., vol. 13, pp. 129-139, 2012, doi: 10.1016/j.jmbbm.2012.04.021. 
[15] D. Perrin et al., "Patient-specific simulation of endovascular repair surgery with tortuous aneurysms requiring flexible stent-grafts," J. Mech. Behav. Biomed. Mater., vol. 63, pp. 86-99, 2016, doi: 10.1016/j.jmbbm.2016.06.013.

[16] J. Gindre et al., "Finite element simulation of the insertion of guidewires during an EVAR procedure: example of a complex patient case, a first step toward patient-specific parameterized models," int. J. Numer. Method. Biomed. Eng., vol. 31, no. 7, p. e02716, Jul. 2015, doi: 10.1002/cnm.2716.

[17] D. Perrin et al., "Deployment of stent grafts in curved aneurysmal arteries: Toward a predictive numerical tool," int. J. Numer. Method. Biomed. Eng., vol. 31, no. 1, pp. 26-36, 2015, doi: 10.1002/cnm.2698.

[18] H. Mohammadi, S. Lessard, E. Therasse, R. Mongrain, and G. Soulez, "A Numerical Preoperative Planning Model to Predict Arterial Deformations in Endovascular Aortic Aneurysm Repair," Ann. Biomed. Eng., vol. 46, no. 12, pp. 2148-2161, 2018, doi: 10.1007/s10439-018-2093-8.

[19] A. Hemmler, B. Lutz, C. Reeps, G. Kalender, and M. W. Gee, "A methodology for in silico endovascular repair of abdominal aortic aneurysms," Biomech. Model. Mechanobiol., vol. 17, no. 4, pp. 1139-1164, 2018, doi: 10.1007/s10237-018-1020-0.

[20] F. Auricchio, M. Conti, S. Marconi, A. Reali, J. L. Tolenaar, and S. Trimarchi, "Patient-specific aortic endografting simulation: From diagnosis to prediction," Comput. Biol. Med., vol. 43, no. 4, pp. 386394, 2013, doi: 10.1016/j.compbiomed.2013.01.006.

[21] R. M. Sanford, S. A. Crawford, H. Genis, M. G. Doyle, T. L. Forbes, and C. H. Amon, "Predicting Rotation in Fenestrated Endovascular Aneurysm Repair Using Finite Element Analysis," J. Biomech. Eng., vol. 140, no. 9, pp. 1-8, 2018, doi: 10.1115/1.4040124.

[22] S. A. Crawford, R. M. Sanford, M. G. Doyle, M. Wheatcroft, C. H. Amon, and T. L. Forbes, "Prediction of advanced endovascular stent graft rotation and its associated morbidity and mortality," J. Vasc. Surg., vol. 68, no. 2, pp. 348-355, 2018, doi: 10.1016/j.jvs.2017.11.061.

[23] A. Kaladji, A. Lucas, G. Kervio, P. Haigron, and A. Cardon, "Sizing for endovascular aneurysm repair: Clinical evaluation of a new automated three-dimensional software," Ann. Vasc. Surg., vol. 24, no. 7, pp. 912-920, 2010, doi: 10.1016/j.avsg.2010.03.018.

[24] A. Gray, Modern Differential Geometry of Curves and Surfaces with Mathematica, 2nd ed., CRC Press. 1997.

[25] F. Auricchio and R. L. Taylor, "Shape-memory alloys: macromodelling and numerical simulations of the superelastic behavior," Comput. Methods Appl. Mech. Eng., vol. 143, no. 3-4, pp. 175-194, 1997, doi: 10.1016/S0045-7825(96)01232-7.

[26] N. Demanget et al., "Computational comparison of the bending behavior of aortic stent-grafts," J. Mech. Behav. Biomed. Mater., vol. 5, no. 1, pp. 272-282, 2012, doi: 10.1016/j.jmbbm.2011.09.006.

[27] C. Kleinstreuer, Z. Li, C. A. Basciano, S. Seelecke, and M. A. Farber, "Computational mechanics of Nitinol stent grafts," J. Biomech., vol. 41, no. 11, pp. 2370-2378, 2008, doi: 10.1016/j.jbiomech.2008.05.032.

[28] L. Antiga, M. Piccinelli, L. Botti, B. Ene-lordache, A. Remuzzi, and D. A. Steinman, "An image-based modeling framework for patient-specific computational hemodynamics," Med. Biol. Eng. Comput., vol. 46, no. 11, pp. 1097-1112, 2008, doi: 10.1007/s11517-008-0420-1.

[29] D. Roy, S. Lerouge, K. Inaekyan, C. Kauffmann, R. Mongrain, and G. Soulez, "Experimental validation of more realistic computer models for stent-graft repair of abdominal aortic aneurysms, 
including pre-load assessment," int. J. Numer. Method. Biomed. Eng., vol. 32, no. 12, p. e02769, 2016, doi: 10.1002/cnm.2769.

[30] D. K. Liang, D. Z. Yang, M. Qi, and W. Q. Wang, "Finite element analysis of the implantation of a balloon-expandable stent in a stenosed artery," Int. J. Cardiol., vol. 104, no. 3, pp. 314-318, 2005, doi: 10.1016/j.ijcard.2004.12.033.

[31] L. H. Timmins, C. A. Meyer, M. R. Moreno, and J. E. Moore, "Effects of Stent Design and Atherosclerotic Plaque Composition on Arterial Wall Biomechanics," J. Endovasc. Ther., vol. 15, no. 6, pp. 643-654, 2008, doi: 10.1583/08-2443.1.

[32] D. E. Kiousis, S. F. Rubinigg, M. Auer, and G. A. Holzapfel, "A Methodology to Analyze Changes in Lipid Core and Calcification Onto Fibrous Cap Vulnerability: The Human Atherosclerotic Carotid Bifurcation as an Illustratory Example," J. Biomech. Eng., vol. 131, no. 12, p. 121002, 2009, doi: 10.1115/1.4000078.

[33] D. Li, A. M. Robertson, G. Lin, and M. Lovell, "Finite element modeling of cerebral angioplasty using a structural multi-mechanism anisotropic damage model," Int. J. Numer. Methods Eng., vol. 92, no. 5, pp. 457-474, 2012, doi: 10.1002/nme.

[34] F. Auricchio, M. Conti, M. De Beule, G. De Santis, and B. Verhegghe, "Carotid artery stenting simulation: From patient-specific images to finite element analysis," Med. Eng. Phys., vol. 33, no. 3, pp. 281-289, 2011, doi: 10.1016/j.medengphy.2010.10.011.

[35] P. Mortier et al., "A Novel Simulation Strategy for Stent Insertion and Deployment in Curved Coronary Bifurcations: Comparison of Three Drug-Eluting Stents," Ann. Biomed. Eng., vol. 38, no. 1, pp. 88-99, 2010, doi: 10.1007/s10439-009-9836-5.

[36] M. Conti et al., "Patient-specific finite element analysis of popliteal stenting," Meccanica, vol. 52, no. 3, pp. 633-644, 2017, doi: 10.1007/s11012-016-0452-9.

[37] C. Gökgöl, N. Diehm, F. R. Nezami, and P. Büchler, "Nitinol Stent Oversizing in Patients Undergoing Popliteal Artery Revascularization: A Finite Element Study," Ann. Biomed. Eng., vol. 43, no. 12, pp. 2868-2880, 2015, doi: 10.1007/s10439-015-1358-8.

[38] J. Gindre et al., "Patient-Specific Finite-Element Simulation of the Insertion of Guidewire during an EVAR Procedure: Guidewire Position Prediction Validation on 28 Cases," IEEE Trans. Biomed. Eng., vol. 64, no. 5, pp. 1057-1066, 2017, doi: 10.1109/TBME.2016.2587362.

[39] F. Cochennec, J. P. Becquemin, P. Desgranges, E. Allaire, H. Kobeiter, and F. Roudot-Thoraval, "Limb Graft Occlusion Following EVAR: Clinical Pattern, Outcomes and Predictive Factors of Occurrence," Eur. J. Vasc. Endovasc. Surg., vol. 34, no. 1, pp. 59-65, 2007, doi: 10.1016/j.ejvs.2007.01.009.

[40] G. Maleux, D. Ph, M. Koolen, and S. Heye, "Complications after Endovascular Aneurysm Repair," Semin. Intervent. Radiol., vol. 26, no. 1, pp. 3-9, 2009, doi: 10.1055/s-0029-1208377. 